\title{
Parametrically Excited Oscillations of Second-Order Functional Differential Equations and Application to Duffing Equations with Time Delay Feedback
}

\author{
Mervan Pašić \\ Department of Applied Mathematics, Faculty of Electrical Engineering and Computing, \\ University of Zagreb, 10000 Zagreb, Croatia \\ Correspondence should be addressed to Mervan Pašić; mervan.pasic@gmail.com \\ Received 8 December 2013; Accepted 12 February 2014; Published 16 April 2014 \\ Academic Editor: Zhengqiu Zhang \\ Copyright (C) 2014 Mervan Pašić. This is an open access article distributed under the Creative Commons Attribution License, which \\ permits unrestricted use, distribution, and reproduction in any medium, provided the original work is properly cited.

\begin{abstract}
We study oscillatory behaviour of a large class of second-order functional differential equations with three freedom real nonnegative parameters. According to a new oscillation criterion, we show that if at least one of these three parameters is large enough, then the main equation must be oscillatory. As an application, we study a class of Duffing type quasilinear equations with nonlinear time delayed feedback and their oscillations excited by the control gain parameter or amplitude of forcing term. Finally, some open
\end{abstract} \\ questions and comments are given for the purpose of further study on this topic.
}

\section{Introduction}

Let $\lambda, \mu, \rho$ be three nonnegative parameters and let $A(v)$, $B(t, u, v), F(t, u), G(t, u)$, and $e(t)$ be continuous functions in all their variables satisfying some conditions determined in Section 2. We consider the following large class of secondorder functional differential equations:

$$
\begin{gathered}
\left(r(t) A\left(x^{\prime}(t)\right)\right)^{\prime}+B\left(t, x(t), x^{\prime}(t)\right)+\lambda F(t, x(\tau(t))) \\
+\mu G(t, x(\sigma(t)))=\rho e(t), \quad t \geq t_{0},
\end{gathered}
$$

where as usual the functional terms $\tau(t)$ and $\sigma(t)$ satisfy $\tau(t) \leq$ $t, \lim _{t \rightarrow \infty} \tau(t)=\infty$, and $\sigma(t) \geq t$. Three cases are studied simultaneously: delay $(\tau(t) \neq \equiv t, \sigma(t)=t)$, advanced $(\tau(t)=t$, $\sigma(t) \neq \equiv t)$, and delay-advanced $(\tau(t) \neq \equiv t, \sigma(t) \not \equiv t)$, and both $\tau(t)$ and $\sigma(t)$ are increasing functions.

A continuous function $x(t)$ is called nonoscillatory if there is a point $T \geq t_{0}$ such that $x(t) \neq 0$ for all $t \geq$ $T$. Otherwise, $x(t)$ is an oscillatory function. A function $x=x(t), x \in C^{2}\left(\left(t_{0}, \infty\right), \mathbb{R}\right)$, is called the (extendable) solution of (1) if it satisfies equality in (1) for all $t>t_{0}$. Equation (1) is oscillatory if all its solutions are oscillatory.
In the paper, we investigate conditions on $F(t, u), G(t, u)$, and $e(t)$ under which (1) is oscillatory provided at least one of parameters $\lambda, \mu$, and $\rho$ is large enough. It is shortly called the parametrically excited oscillations of (1). In Section 4 we discuss some known oscillation criteria published in [1-9] which allow the parametrically excited oscillations but only in the form of examples. On various problems concerning the functional differential equations we refer the reader to [10-15] and the references therein.

In Section 2 we state a fundamental lemma proposing a new oscillation criterion that plays a crucial role in the formulation of the main results illustrated on some suitable chosen examples. In Section 3 we consider an application of the main results to the Duffing type quasilinear equations with time delayed feedback, taking into account the known results in applied sciences concerning such kind of nonlinear oscillators without time delay, see [16-37], and with time delay, see [38-48] and the references therein. In Section 5 we present some open questions and comments for further study that can follow our main results. And in Section 6, we describe the method for proving the main results of the paper. 


\section{Main Assumptions and Results}

Let $\mathbb{R}_{+}=(0, \infty)$. For the functions $r=r(t)$ and $A=A(v)$ both appearing in the second-order differential operator of (1), we suppose the following:

$$
0<r(t) \leq r_{0} \text { on }\left[t_{0}, \infty\right), \quad r \in C^{1}\left(\left[t_{0}, \infty\right), \mathbb{R}_{+}\right),
$$

and $A(v)$ is odd, increasing, and

$$
|u|^{-p-1} A(v) v \geq \alpha\left(|A(v)||u|^{-p}\right)
$$

for some $p>0$ and all $u \neq 0, v \in \mathbb{R}$,

$$
\begin{aligned}
\alpha= & \alpha(s), \quad \alpha \in C^{1}([0, \infty),[0, \infty)), \\
& \frac{1}{1+\alpha} \in L^{1}\left(\mathbb{R}_{+}, \mathbb{R}_{+}\right), \quad \pi_{*}=\int_{0}^{\infty} \frac{2 d s}{1+\alpha(s)},
\end{aligned}
$$

$\alpha(c s) \geq c^{\gamma} \alpha(s)$ for some $\gamma \geq 1$ and all $c>0, s \geq 0$.

For instance, it is simple to check that for $\alpha(s)=s^{\gamma}$, hypothesis (3) is fulfilled in the next three most important cases of $A(v)$ : the linear operator $A(v)=v$ if $p=1$ and $\gamma=2$, the quasilinear $p$-Laplacian operator $A(v)=|v|^{p-1} v$ if $p \geq 1$ and $\gamma=(p+1) / p$, and the quasilinear mean curvature operator $A(v)=v\left(1+v^{2}\right)^{-1 / 2}$ if $p=1$ and $\gamma=2$. Some details about the number $\pi_{*}$ which depends on the function $\alpha(s)$ given in (3) are presented in Section 6.

The damped term $B=B(t, u, v)$ satisfies the strong condition

$$
B(t, u, v) \operatorname{sgn}(u) \geq 0 \quad \forall t \geq t_{0}, u \neq 0, v \in \mathbb{R} .
$$

We hope that (4) can be relaxed with some weaker condition, which is commented as an open problem in Section 5 below.

In the following fundamental lemma which plays a crucial role in the proof of the main results, we are working with such solutions $x(t)$ of (1) that satisfies the inequality

$$
\begin{aligned}
& {[\lambda F(t, x(\tau(t)))+\mu G(t, x(\sigma(t)))-\rho e(t)]} \\
& \quad \times\left(|x(t)|^{p-1} x(t)\right)^{-1} \geq k(\lambda, \mu, \rho) Q(t),
\end{aligned}
$$

for all $t \in J$ and some interval $J$, where the functions $k(\lambda, \mu, \rho)$ and $Q(t)$ do not depend on $x(t)$ but only on $F, G, e$ and they are determined in the process below. The functions $k(\lambda, \mu, \rho)$ and $Q(t)$ present the key point in the parametrically excited oscillations.

Lemma 1. Let assumptions (2), (3), and (4) hold. Let ( $a, b)$ and $(c, d)$ be two disjoint open intervals such that $\sigma(b) \leq \tau(c)$. Let the functions $k=k(\lambda, \mu, \rho), k \in C\left([0, \infty)^{3}, \mathbb{R}_{+}\right)$, and $Q=$ $Q(t), Q \in C(\bar{J},[0, \infty)), Q(t) \not \equiv 0$ on $J$, be such that

$$
\frac{p^{1 / \gamma}}{r_{0}^{1-1 / \gamma}} \frac{[k(\lambda, \mu, \rho)]^{1-1 / \gamma}}{\pi_{*}} \int_{J} Q(t) d t \geq\left(\max _{t \in \bar{J}} Q(t)\right)^{1 / \gamma},
$$

for both $J=(a, b)$ and $J=(c, d)$, for all $\lambda \geq \lambda_{0}, \mu \geq \mu_{0}$, and $\rho \geq \rho_{0}$, and for some $\left(\lambda_{0}, \mu_{0}, \rho_{0}\right) \in \mathbb{R}_{+}^{3}$, where $r_{0}, p, \gamma$, and $\pi_{*}$ are constants defined, respectively, in (2) and (3). Let $x(t)$ be a solution of (1) satisfying the next two statements:

$$
\text { if } \begin{array}{r}
x(t)>0, \quad x(\tau(t))>0, \quad x(\sigma(t))>0 \\
\text { on }(\tau(a), \sigma(b)) ;
\end{array}
$$

then $x(t)$ satisfies $(5)$ on $(a, b)$,

$$
\begin{aligned}
& \text { if } x(t)<0, \quad x(\tau(t))<0, \quad x(\sigma(t))<0 \\
& \text { on }(\tau(c), \sigma(d)) \text {, }
\end{aligned}
$$

then $(t)$ satisfies (5) on $(c, d)$.

Then $x(t)$ has at least one zero point in $(\tau(\tau(a)), \sigma(\sigma(d)))$.

This lemma simultaneously holds for all three types of functional arguments: delay, advanced, and delay-advanced. It will be proved in Section 6. In Corollaries 9, 10, and 11 below, we give some simple conditions on two functions $f(t), g(t)$ and two numbers $p_{1}, p_{2}$ such that all solutions of (1) with the functions $F(t, u)=f(t, u)|u|^{p_{1}} \operatorname{sgn}(u)$ and $G(t, u)=g(t, u)|u|^{p_{2}} \operatorname{sgn}(u)$ satisfy required statements (7) and (8) with respect to some intervals $\left(a_{n}, b_{n}\right)$ and the explicitly given functions $k(\lambda, \mu, \rho)$ and $Q_{n}(t)$ satisfying (6), where $e(t)$ satisfies a basic assumption.

In what follows, $\left(a_{n}, b_{n}\right), n \in \mathbb{N}$, denotes a sequence of disjoint open intervals such that $t_{0} \leq \tau\left(a_{n}\right)<\sigma\left(b_{n}\right) \leq$ $\tau\left(a_{n+1}\right)<\sigma\left(b_{n+1}\right) \leq \cdots$, and $a_{n} \rightarrow \infty$ as $n \rightarrow \infty$. Now we present several variations of Lemma 1 in which essential inequality (6) is relaxed with some asymptotic assumptions that are simpler to be verified in several applications.

Lemma 2. Let assumptions (2), (3), and (4) hold. Let the continuous function $k=k(\lambda, \mu, \rho)>0$ and the sequence of functions $Q_{n}=Q_{n}(t), Q_{n} \in C\left(\left[a_{n}, b_{n}\right],[0, \infty)\right), Q_{n}(t) \not \equiv 0$ on $\left(a_{n}, b_{n}\right)$, satisfy the next two inequalities: there are constants $c_{0}>0, n_{0} \in \mathbb{N}$, and $\left(\lambda_{0}, \mu_{0}, \rho_{0}\right) \in \mathbb{R}_{+}^{3}$ such that

$$
\begin{gathered}
k(\lambda, \mu, \rho) \geq r_{0} p^{-1 /(\gamma-1)}\left(\frac{2 \pi_{*}}{c_{0}}\right)^{\gamma /(\gamma-1)} \\
\forall \lambda \geq \lambda_{0}, \mu \geq \mu_{0}, \rho \geq \rho_{0},
\end{gathered}
$$

where the numbers $r_{0}, p, \gamma$, and $\pi_{*}$ are, respectively, from (2) and (3), and

$$
\lim _{n \rightarrow \infty}\left(\frac{1}{\left(\max _{t \in\left[a_{n}, b_{n}\right]} Q_{n}(t)\right)^{1 / \gamma}} \int_{a_{n}}^{b_{n}} Q_{n}(t) d t\right) \geq c_{0}>0 .
$$

If $x(t)$ is a solution of (1) that satisfies (7) and (8) with $a=$ $a_{2 n-1}, b=b_{2 n-1}, c=a_{2 n}, d=b_{2 n}$, and $Q(t)=Q_{n}(t)$, then $x(t)$ has at least one zero point in $\left(\tau\left(\tau\left(a_{2 n-1}\right)\right), \sigma\left(\sigma\left(b_{2 n}\right)\right)\right), \forall n \geq n_{0}$.

In the following slightly simpler version of Lemma 2, inequality (9) is replaced with an asymptotic condition and, at the same time, the limit in (10) is relaxed with the limit inferior. Thus, conditions (9) and (10) are replaced with more practical ones. 
Lemma 3. Let assumptions (2), (3), and (4) hold. Let the continuous function $k=k(\lambda, \mu, \rho)>0$ and the sequence of functions $Q_{n}=Q_{n}(t), Q_{n} \in C\left(\left[a_{n}, b_{n}\right],[0, \infty)\right), Q_{n}(t) \not \equiv 0$, on $\left(a_{n}, b_{n}\right)$ satisfy, respectively,

$$
\begin{gathered}
k(\lambda, \mu, \rho) \longrightarrow \infty \text { as } \lambda \longrightarrow \infty \\
\text { or } \mu \longrightarrow \infty \text { or } \rho \longrightarrow \infty, \\
\lim \inf _{n \rightarrow \infty}\left(\frac{1}{\left(\max _{t \in\left[a_{n}, b_{n}\right]} Q_{n}(t)\right)^{1 / \gamma}} \int_{a_{n}}^{b_{n}} Q_{n}(t) d t\right)>0 .
\end{gathered}
$$

If $x(t)$ is a solution of (1) that satisfies (7) and (8) with $a=$ $a_{2 n-1}, b=b_{2 n-1}, c=a_{2 n}, d=b_{2 n}$, and $Q(t)=Q_{n}(t)$, then $x(t)$ has at least one zero point in $\left(\tau\left(\tau\left(a_{2 n-1}\right)\right), \sigma\left(\sigma\left(b_{2 n}\right)\right)\right), \forall n \geq n_{0}$, and for some $n_{0} \in \mathbb{N}$.

In some concrete cases, we use the next version of Lemmas 2 and 3, where condition (10) or (12) is replaced with appropriate one that appears in (1) with periodic coefficients.

Lemma 4. Let assumptions (2), (3), and (4) hold. Let the continuous function $k=k(\lambda, \mu, \rho)>0$ and the sequence of functions $Q_{n}=Q_{n}(t), Q_{n} \in C\left(\left[a_{n}, b_{n}\right],[0, \infty)\right), Q_{n}(t) \not \equiv 0$, on $\left(a_{n}, b_{n}\right)$ satisfy, respectively, (11) and, for some $C_{0}, c_{1} \in \mathbb{R}$,

$$
\begin{array}{r}
0<\max _{t \in\left[a_{n}, b_{n}\right]} Q_{n}(t) \leq C_{0}, \quad \int_{a_{n}}^{b_{n}} Q_{n}(t) d t \geq c_{1}>0, \\
\forall n \geq n_{0},
\end{array}
$$

and some $n_{0} \in \mathbb{N}$. If $x(t)$ is a solution of (1) that satisfies (7) and (8) with $a=a_{2 n-1}, b=b_{2 n-1}, c=a_{2 n}, d=b_{2 n}$, and $Q(t)=Q_{n}(t)$, then $x(t)$ has at least one zero point in $\left(\tau\left(\tau\left(a_{2 n-1}\right)\right), \sigma\left(\sigma\left(b_{2 n}\right)\right)\right), \forall n \geq n_{0}$.

Next, we suppose that the coefficient $r(t)$ additionally satisfies

$$
\begin{gathered}
r(s) \leq r(t) \text { in delay, } \\
r(s) \geq r(t) \text { in advanced case } \forall s \leq t, \\
r(t) \text { is a constant in delay-advanced case, }
\end{gathered}
$$

and the forcing term $e(t)$ satisfies

$$
\begin{aligned}
& e(t) \leq 0 \text { on }\left[\tau\left(a_{2 n-1}\right), \sigma\left(b_{2 n-1}\right)\right], \\
& e(t) \geq 0 \text { on }\left[\tau\left(a_{2 n}\right), \sigma\left(b_{2 n}\right)\right], \quad n \in \mathbb{N} .
\end{aligned}
$$

We remark that $e(t)$ remains arbitrary function outside the set $\bigcup_{n}\left(\tau\left(a_{n}\right), \sigma\left(b_{n}\right)\right)$.

The first result of the paper deals with delay equation (1).

Theorem 5. Let assumptions (2), (3), (4), (14), and (15) hold, $\tau(t)=t-\tau, \tau \geq 0, \sigma(t) \equiv t, G(t, u) \equiv 0$, and let $F(t, u)$ satisfy

$$
\begin{gathered}
F(t, u) \operatorname{sgn}(u) \geq f(t)|u|^{q} \quad \forall u \neq 0, t \geq t_{0}, \\
f(t) \geq 0, \quad f(t) \not \equiv 0 \text { on }\left[\tau\left(a_{n}\right), b_{n}\right],
\end{gathered}
$$

where $q \geq p$, number $p$ is from (3), sequence $\left(a_{n}, b_{n}\right)$ is from (15), and $f \in C\left(\left[t_{0}, \infty\right), \mathbb{R}\right)$ is a periodic function with period $T_{*}>0$ such that

$$
\begin{aligned}
{\left[a_{2 n-1}+T_{*}, b_{2 n-1}+T_{*}\right] } & \subseteq\left[a_{2 n+1}, b_{2 n+1}\right], \\
{\left[a_{2 n}+T_{*}, b_{2 n}+T_{*}\right] } & \subseteq\left[a_{2 n+2}, b_{2 n+2}\right], \quad n \in \mathbb{N} .
\end{aligned}
$$

Then (1) is oscillatory in the next two cases: $q=p$ and parameter $\lambda$ is large enough; $q>p, \lambda>0, \rho>0$, and at least one of parameters $\lambda$ and $\rho$ is large enough.

The proof of Theorem 5 is presented in Section 6 and it is based on Lemma 4, where

$$
k(\lambda, \mu, \rho)=\lambda^{p / q} \rho^{1-(p / q)}, \quad q \geq p,
$$

$$
\begin{aligned}
& Q_{n}(t) \\
& = \begin{cases}f(t)\left(\frac{t-a_{n}}{t-a_{n}+\tau}\right)^{p}, & \text { if } q=p, \\
\frac{q}{p}\left(\frac{p}{q-p}\right)^{(q / p)-1}|e(t)|^{1-(p / q)}(f(t))^{p / q} & \\
\times\left(\frac{t-a_{n}}{t-a_{n}+\tau}\right)^{p}, & \text { if } q>p .\end{cases}
\end{aligned}
$$

The second result deals with advanced equation (1).

Theorem 6. Let assumptions (2), (3), (4), (14), and (15) hold, $\tau(t) \equiv t, \sigma(t)=t+\sigma, \sigma \geq 0, F(t, u) \equiv 0$, and let $G(t, u)$ satisfy

$$
\begin{gathered}
G(t, u) \operatorname{sgn}(u) \geq g(t)|u|^{q} \quad \forall u \neq 0, t \geq t_{0}, \\
g(t) \geq 0, \quad g(t) \not \equiv 0 \text { on }\left[a_{n}, \sigma\left(b_{n}\right)\right],
\end{gathered}
$$

where $q \geq p$, number $p$ is from (3), sequence $\left(a_{n}, b_{n}\right)$ is from (15), and $g \in C\left(\left[t_{0}, \infty\right), \mathbb{R}\right)$ is a periodic function with period $T_{*}>0$ such that (17) is fulfilled. Then (1) is oscillatory in the next two cases: $q=p$ and parameter $\mu$ is large enough; $q>p$, $\mu>0, \rho>0$, and at least one of parameters $\mu$ and $\rho$ is large enough.

The proof of Theorem 6 is based on Lemma 4 (see Section 6), where

$$
k(\lambda, \mu, \rho)=\mu^{p / q} \rho^{1-(p / q)}, \quad q \geq p
$$

$Q_{n}(t)$

$$
= \begin{cases}g(t)\left(\frac{b_{n}-t}{b_{n}-t+\sigma}\right)^{p}, & \text { if } q=p, \\ \frac{q}{p}\left(\frac{p}{q-p}\right)^{(q / p)-1}|e(t)|^{1-(p / q)}(g(t))^{p / q} & \\ \times\left(\frac{b_{n}-t}{b_{n}-t+\sigma}\right)^{p}, & \text { if } q>p .\end{cases}
$$

The third result deals with delay-advanced equation (1). 
Theorem 7. Let assumptions (2), (3), (4), (14), and (15) hold, $\tau(t)=t-\tau, \tau \geq 0, \sigma(t)=t+\sigma, \sigma \geq 0$, and $F(t, u)$ and $G(t, u)$ satisfy

$$
\begin{gathered}
F(t, u) \operatorname{sgn}(u) \geq f(t)|u|^{p_{1}}, \quad G(t, u) \operatorname{sgn}(u) \geq g(t)|u|^{p_{2}} \\
\forall u \neq 0, t \geq t_{0}, \\
f(t) \geq 0, \quad f(t) \not \equiv 0, \quad g(t) \geq 0, \\
g(t) \not \equiv 0 \text { on }\left[\tau\left(a_{n}\right), \sigma\left(b_{n}\right)\right],
\end{gathered}
$$

where, additionally, $f, g, e \in C\left(\left[t_{0}, \infty\right), \mathbb{R}\right)$ are three periodic functions having a common period $T_{*}>0$ such that (17) is fulfilled, where $e(t)$ is the forcing term in (1). Then (1) is oscillatory provided one of the next two cases is fulfilled, where the number $p$ is from (3): (1) (in superlinear delay-advanced case) $p_{1}>p, p_{2}>p, \rho>0$, and either parameter $\rho$ is large enough or at least one of $\lambda$ and $\mu$ is large enough; (2) (in supersublinear delay-advanced case) $p_{1}>p>p_{2}>0, \lambda>0$, $\mu>0, \rho>0$, and at least one of parameters $\lambda, \mu$, and $\rho$ is large enough.

The proof of Theorem 7 is based on Lemma 4 (see Section 6), where

$$
k(\lambda, \mu, \rho)=\left\{\begin{array}{l}
\min \left\{\lambda^{p / p_{1}} \rho^{1-\left(p / p_{1}\right)}, \mu^{p / p_{2}} \rho^{1-\left(p / p_{2}\right)}\right\}, \\
\quad \text { superlinear case, } \\
\lambda^{\eta_{1}} \mu^{\eta_{1}} \rho^{\eta_{0}}, \\
\text { supersublinear case, }
\end{array}\right.
$$

$$
\begin{aligned}
& Q_{n}(t) \\
& \qquad\left\{\begin{array}{l}
\frac{p_{1}}{p}\left(\frac{p}{2\left(p_{1}-p\right)}\right)^{\left(p_{1} / p\right)-1} \\
\times|e(t)|^{1-\left(p / p_{1}\right)}(f(t))^{p / p_{1}} \tau_{n}(t) \\
+\frac{p_{2}}{p}\left(\frac{p}{2\left(p_{2}-p\right)}\right)^{\left(p_{2} / p\right)-1}|e(t)|^{1-\left(p / p_{2}\right)} \\
\times(g(t))^{p / p_{2}} \sigma_{n}(t), \\
\quad \text { superlinear case, } \\
|e(t)|^{\eta_{0}}(f(t))^{\eta_{1}}(g(t))^{\eta_{2}}\left(\tau_{n}(t)\right)^{\eta_{1}\left(p_{1} / p\right)} \\
\times\left(\sigma_{n}(t)\right)^{\eta_{2}\left(p_{2} / p\right)} \prod_{i=0}^{2} \eta_{i}^{-\eta_{i}}, \\
\quad \text { supersublinear case, }
\end{array}\right.
\end{aligned}
$$

where we denote

$$
\tau_{n}(t)=\left(\frac{t-a_{n}}{t-a_{n}+\tau}\right)^{p}, \quad \sigma_{n}(t)=\left(\frac{b_{n}-t}{b_{n}-t+\sigma}\right)^{p} .
$$

Here, the numbers $\eta_{0}, \eta_{1}, \eta_{2} \in(0,1)$ are chosen such that $\eta_{0}+$ $\eta_{1}+\eta_{2}=1$ and $p_{1} \eta_{1}+p_{2} \eta_{2}=p$. Let us mention that if $p_{1}=5 / 2, p=1$ and $p_{2}=1 / 2$, and $\eta_{0}=\eta_{1}=\eta_{2}=1 / 3$, then $\left(p_{1}, p_{2}\right)$ and $\left(\eta_{0}, \eta_{1}, \eta_{2}\right)$ satisfy previous two equalities. About the existence of such $(N+1)$-tuple $\left(\eta_{0}, \eta_{1}, \ldots, \eta_{N}\right)$ in a general case, we refer to [49].
Remark 8. A difference between assumptions of Theorems 5, 6 , and 7 is that $e(t)$ in Theorems 5 and 6 is not necessarily periodic or bounded function as it is supposed in Theorem 7 .

Now, we study an important class of second-order functional differential equations as a particular case of (1):

$$
\begin{aligned}
& \left(r(t) A\left(x^{\prime}(t)\right)\right)^{\prime}+B\left(t, x(t), x^{\prime}(t)\right) \\
& \quad+\lambda f(t)|x(\tau(t))|^{p_{1}} \operatorname{sgn}(x(\tau(t))) \\
& \quad+\mu g(t)|x(\sigma(t))|^{p_{2}} \operatorname{sgn}(x(\sigma(t)))=\rho e(t), \quad t \geq t_{0},
\end{aligned}
$$

where $\tau(t)=t-\tau, \sigma(t)=t+\sigma$, and $\lambda, \mu, \rho, \tau, \sigma \geq 0$ and $p_{1}, p_{2}>0$. Using previous theorems, we are able to state the main consequences showing the parametrically excited oscillations in (27).

Corollary 9 (delay equation). Let assumptions (2), (3), (4), (14), and (15) hold. Let $g(t) \equiv 0, f(t) \geq 0$, and $f(t) \neq 0$ on $\left[a_{n}-\tau, b_{n}\right]$, where $f \in C\left(\left[t_{0}, \infty\right), \mathbb{R}\right)$ is a periodic function with period $T^{*}$ satisfying (16). Equation (27) is oscillatory in the following two cases: $p_{1}=p$ and parameter $\lambda$ is large enough; $p_{1}>p, \lambda>0, \rho>0$, and at least one of parameters $\lambda$ and $\rho$ is large enough.

Corollary 10 (advanced equation). Let assumptions (2), (3), (4), (14), and (15) hold. Let $f(t) \equiv 0, g(t) \geq 0$, and $g(t) \neq \equiv$ on $\left[a_{n}, b_{n}+\sigma\right]$, where $g \in C\left(\left[t_{0}, \infty\right), \mathbb{R}\right)$ is a periodic function with period $T^{*}$ satisfying (17). Then (27) is oscillatory in the following two cases: $p_{2}=p$ and parameter $\mu$ is large enough; $p_{2}>p, \mu>0, \rho>0$, and at least one of parameters $\mu$ and $\rho$ is large enough.

Corollary 11 (delay-advanced equation). Let assumptions (2), (3), (4), (14), and (15) hold, and $f(t)$ and $g(t)$ satisfy

$$
\begin{array}{r}
f(t) \geq 0, \quad f(t), \not \equiv 0 \\
g(t) \geq 0, \quad g(t) \not \equiv 0 \\
\text { on }\left[a_{n}-\tau, b_{n}+\sigma\right],
\end{array}
$$

where, additionally, $f, g, e \in C\left(\left[t_{0}, \infty\right), \mathbb{R}\right)$ are three periodic functions having a common period $T_{*}>0$ such that (17) is fulfilled, where $e(t)$ is the forcing term in (1). Then (27) is oscillatory in the next two cases, where the number $p$ is from (3): (1) (superlinear case) $p_{1}>p, p_{2}>p, \rho>0$, and either parameter $\rho$ is large enough or at least one of $\lambda$ and $\mu$ is large enough; (2) (supersublinear case) $p_{1}>p>p_{2}>0, \lambda>0$, $\mu>0, \rho>0$, and at least one of parameters $\lambda, \mu$, and $\rho$ is large enough.

According to previous corollaries, we can derive the following examples.

Example 12 (delay case). Let $p \geq 1, \varepsilon \geq 0$, and $m \in \mathbb{N}$ be fixed and $\tau_{m} \in \mathbb{R}, 0 \leq \tau_{m}<\pi /(4 m)$. With the help of 
Corollary 9, the following two different classes of quasilinear delay differential equations:

$$
\begin{aligned}
& \left(\left|x^{\prime}(t)\right|^{p-1} x^{\prime}(t)\right)^{\prime}+\lambda \sin (m t)\left|x\left(t-\tau_{m}\right)\right|^{p+\varepsilon} \\
& \times \operatorname{sgn}\left(x\left(t-\tau_{m}\right)\right)=-\rho \cos (2 m t), \\
& \left(\frac{x^{\prime}(t)}{\sqrt{1+x^{\prime 2}(t)}}\right)^{\prime}+\lambda \cos (m t)\left|x\left(t-\tau_{m}\right)\right|^{1+\varepsilon} \\
& \times \operatorname{sgn}\left(x\left(t-\tau_{m}\right)\right)=-\rho \cos (2 m t),
\end{aligned}
$$

are oscillatory provided at least one of $\lambda>0$ and $\rho>0$ is large enough (the case $\rho=0$ is possible if $\varepsilon=0$ ). It is because, for all $n \in \mathbb{N}$, we have

$$
\begin{aligned}
& -\cos (2 m t)\left\{\begin{array}{l}
\leq 0 \text { on }\left[\frac{2 n \pi}{m}, \frac{2 n \pi}{m}+\frac{\pi}{4 m}\right], \\
\geq 0 \text { on }\left[\frac{2 n \pi}{m}+\frac{\pi}{4 m}, \frac{2 n \pi}{m}+\frac{\pi}{2 m}\right],
\end{array}\right. \\
& \sin (m t) \geq 0, \quad \cos (m t) \geq 0 \text { on }\left[\frac{2 n \pi}{m}, \frac{2 n \pi}{m}+\frac{\pi}{2 m}\right], \\
& {\left[\frac{2 n \pi}{m}+\tau_{m}+T_{*}, \frac{2 n \pi}{m}+\frac{\pi}{4 m}+T_{*}\right]} \\
& =\left[\frac{(2 n+2) \pi}{m}+\tau_{m}, \frac{(2 n+2) \pi}{m}+\frac{\pi}{4 m}\right], \\
& {\left[\frac{2 n \pi}{m}+\frac{\pi}{4 m}+\tau_{m}+T_{*}, \frac{2 n \pi}{m}+\frac{\pi}{2 m}+T_{*}\right]} \\
& =\left[\frac{(2 n+2) \pi}{m}+\frac{\pi}{4 m}+\tau_{m}, \frac{(2 n+2) \pi}{m}+\frac{\pi}{2 m}\right],
\end{aligned}
$$

where $T_{*}=2 \pi / m$ is the common period of the functions $\sin (m t)$ and $\cos (m t)$. Thus, in order to apply Corollary 9 , we can choose $a_{2 n-1}:=2 n \pi / m+\tau_{m}, b_{2 n-1}:=2 n \pi / m+\pi /(4 m)$, $a_{2 n}:=2 n \pi / m+\pi /(4 m)+\tau_{m}$, and $b_{2 n}:=2 n \pi / m+\pi /(2 m)$.

Example 13 (advanced case). Let $p \geq 1, \varepsilon \geq 0$, and $m \in$ $\mathbb{N}$ be fixed and $\sigma_{m} \in \mathbb{R}, 0 \leq \sigma_{m}<\pi /(4 m)$. With the help of Corollary 10, the following two classes of quasilinear advanced differential equations:

$$
\begin{aligned}
& \left(\left|x^{\prime}(t)\right|^{p-1} x^{\prime}(t)\right)^{\prime}+\mu \sin (m t)\left|x\left(t+\sigma_{m}\right)\right|^{p+\varepsilon} \\
& \times \operatorname{sgn}\left(x\left(t+\sigma_{m}\right)\right)=-\rho \cos (2 m t), \\
& \left(\frac{x^{\prime}(t)}{\sqrt{1+x^{\prime 2}(t)}}\right)^{\prime}+\mu \cos (m t)\left|x\left(t+\sigma_{m}\right)\right|^{1+\varepsilon} \\
& \quad \times \operatorname{sgn}\left(x\left(t+\sigma_{m}\right)\right)=-\rho \cos (2 m t),
\end{aligned}
$$

are oscillatory provided at least one of $\mu>0$ and $\rho>0$ is large enough (the case $\rho=0$ is possible if $\varepsilon=0$ ). In order to apply Corollary 10, we can choose $a_{2 n-1}:=2 n \pi / m, b_{2 n-1}:=$ $2 n \pi / m+\pi /(4 m)-\sigma_{m}, a_{2 n}:=2 n \pi / m+\pi /(4 m)$, and $b_{2 n}:=$ $2 n \pi / m+\pi /(2 m)-\sigma_{m}$.
Example 14 (delay-advanced case). Let $p \geq 1, \varepsilon_{1}>0, \varepsilon_{2}>0$, and $m \in \mathbb{N}$ be fixed and $\tau_{m} \geq 0$ and $\sigma_{m} \geq 0,0 \leq \tau_{m}+\sigma_{m}<$ $\pi /(4 m)$. With the help of Corollary 11, the following class of quasilinear delay-advanced differential equations:

$$
\begin{aligned}
& \left(\left|x^{\prime}(t)\right|^{p-1} x^{\prime}(t)\right)^{\prime}+\lambda \sin (m t) \\
& \quad \times\left|x\left(t-\tau_{m}\right)\right|^{p+\varepsilon_{1}} \operatorname{sgn}\left(x\left(t-\tau_{m}\right)\right) \\
& \quad+\mu \cos (m t)\left|x\left(t+\sigma_{m}\right)\right|^{p+\varepsilon_{2}} \\
& \quad \times \operatorname{sgn}\left(x\left(t+\sigma_{m}\right)\right)=-\rho \cos (2 m t),
\end{aligned}
$$

is oscillatory provided either $\rho>0$ is large enough or at least one of $\lambda>0$ and $\mu>0$ is large enough. In order to apply Corollary 11, we can choose $a_{2 n-1}:=2 n \pi / m+\tau_{m}, b_{2 n-1}:=$ $2 n \pi / m+\pi /(4 m)-\sigma_{m}, a_{2 n}:=2 n \pi / m+\pi /(4 m)+\tau_{m}$, and $b_{2 n}:=2 n \pi / m+\pi /(2 m)-\sigma_{m}$.

\section{Application to Duffing Equations with Time Delay Feedback}

Let $\lambda \geq 0$ denote the control gain parameter (often called "displacement feedback coefficient"), $\tau>0$ the time delay, and $\rho \geq 0$ and $\omega>0$ the amplitude and frequency of the external force, respectively. Let the function $\Phi=\Phi(t, u)$ that will appear in the delay feedback term $\Phi(t, x(t-\tau))$ satisfy the general condition

$$
\begin{gathered}
\Phi(t, u) \operatorname{sgn}(u) \geq \phi_{0}|u|^{q} \quad \forall t \geq t_{0} \\
u \neq 0 \text { and some } q \geq 1, \phi_{0}>0 .
\end{gathered}
$$

For instance, $\Phi(t, u)=u^{2 m-1}, m \in \mathbb{N}$, or more general, $\Phi(t, u)=\sum_{k=1}^{m} \phi_{k} u^{2 k-1}, \phi_{k}>0, m \in \mathbb{N}$.

In this section, we consider the following large class of undamped possible nonautonomous and nonconservative Duffing equations without or with the general time delay feedback $\Phi(t, x(t-\tau))$ :

$$
\begin{aligned}
& \left(\left|x^{\prime}\right|^{p-1} x^{\prime}\right)^{\prime}+\omega_{0}^{2} x+\frac{\mu_{1}|x|^{r_{1}} \operatorname{sgn}(x)}{\left(\mu_{2}+\mu_{3} x^{2}\right)^{r_{2}}} \\
& +\sum_{i=1}^{m} \beta_{i}(t)|x|^{\alpha_{i}-1} x+\lambda \Phi(t, x(t-\tau))=\rho \cos (\omega t),
\end{aligned}
$$

where $\omega_{0}$ is the natural frequency, $\mu_{1} \geq 0$ is the density of the nonlinear potential (or rigidity coefficient), and $\mu_{2}, \mu_{3}, r_{1}, r_{2}$ are nonnegative constants, $\beta_{i}(t) \geq 0$ and $\alpha_{i} \geq 1$.

When $p=1, \lambda=0$, and $\beta_{i}(t) \equiv \beta_{i}=$ const., (34) contains many most important classes of undamped autonomous Duffing oscillators such as the following:

(i) the strongly nonlinear Duffing oscillator with smooth odd nonlinearity is given in (34) provided $\mu_{1}=0$ and $\alpha_{i}=2 i+1$; let us recall some of its known particular cases:

(a) the classic Duffing oscillator $x^{\prime \prime}+\omega_{0}^{2} x+\beta x^{3}=0$ has been recently studied in the searching of 
solitary wave solutions of classic and generalized Zakharov equations of plasma physics (see [16]) and of nonlinear Schrödinger equation (see [17]); also, it is strongly connected with the Jacobi elliptic equation (see [18]);

(b) the cubic-quintic oscillator $x^{\prime \prime}+\omega_{0}^{2} x+\beta_{1} x^{3}+$ $\beta_{2} x^{5}=0$ is used as a model for the nonlinear dynamics of a slender elastica (see [19]) in nonlinear wave systems (see [20]) for the propagation of a short electromagnetic pulse in a nonlinear medium (see [21]) and in the unimodal Duffing temporal problem (see [22]);

(c) the cubic truly nonlinear oscillator $x^{\prime \prime}+\beta x^{3}=$ 0 models the motion of a ball bearing that oscillates in a glass tube that is bent into a curve (see [23]) as well as the motion of a mass attached to identical stretched elastic wires (see [24]);

(d) the nonhomogeneous Duffing oscillator $x^{\prime \prime}+$ $\omega_{0}^{2} x+\beta x^{3}=\rho \cos (\omega t)$ describes various forced vibrations of beams, springs with nonlinear stiffness, cables, plates, shells, and optical fibres in electrical circuits, in nonlinear isolators, and so forth (see, for instance, $[25,26])$;

(ii) the general Duffing-harmonic oscillator (with rational or irrational nonlinear restoring-force) is given in (30) if $\mu_{1} \neq 0, \beta_{i}=0$, and $\rho=0$; the most known subclasses of these oscillators are

(a) the classic Duffing-harmonic oscillator $x^{\prime \prime}+$ $\left(\mu_{1} x^{3} /\left(\mu_{2}+\mu_{3} x^{2}\right)\right)=0$ which models many conservative nonlinear oscillatory systems; see [27];

(b) the relativistic harmonic oscillator $x^{\prime \prime}+\left(\mu_{1} x / \sqrt{1+x^{2}}\right)=0 ;$ see [28];

(c) the nonlinear oscillator $x^{\prime \prime}+x-\left(\mu_{1} x / \sqrt{1+x^{2}}\right)=$ $0, \mu_{1} \in[0,1]$, which is typified as a mass attached to a stretched elastic wire; see [29, 30];

(d) the nonlinear oscillator $x^{\prime \prime}+$ $\left(\mu_{1} x /\left(\sqrt{\left(1+x^{2}\right)^{3}}\right)=0\right.$ which presents nonlinear oscillations of a punctual charge in the electric field of charged ring; see [31].

Finding several explicit forms of periodic approximate solutions for these oscillators has been intensively studied last years by many authors; see, for instance, [28, 30, 32-37] and also the references therein.

When $\lambda \neq 0$ and linear time delay feedback $\Phi(t, x(t-\tau))=$ $x(t-\tau)$, the following topics have been studied for various types of Duffing oscillators with time delayed feedback: in [38] authors constructed a low-order approximate solution under weak feedback gain parameter; about the low- and high-order approximations see also [39]; in [40] with $\rho=0$, the Hopf bifurcation diagrams have been explored for the approximate periodic solutions (amplitude versus time delay $\tau$ and feedback gain $\lambda$ versus time delay $\tau$ ); moreover, in [41] authors made an analysis on the effect of the control gain and time delay parameters on the amplitude of approximate period solution from the theoretical and numerical points of view; see also [42]; in [43] authors studied the chaotic behaviour with respect to gains and time delay parameters; see also [44].

Equations under time delay control such as (34) (especially with damped term) are used as a model for various controlled physical, mechanical, and engineering systems with time delays; see, for instance, [39, 45-48] and the references therein.

Here, (34) contains very general nonlinear time delay feedback $\Phi(t, x(t-\tau))$ with $\Phi$ satisfying (33) and the linear time delay feedback $x(t-\tau)$ is only a particular case of it, and, to the best of our knowledge, the previous topics are not considered for (34), as yet. Moreover, with such an $\Phi$, the oscillations of (34) can be taken under a doubt even with the linear time delay feedback (see the nature of the approximations given in $[38,39])$. Hence, we can pose the following question: under what conditions on equation's parameters, (34) is a nonlinear oscillator, that is, possesses only oscillatory solutions? An answer is given in the next result as an easy consequence of the parametrically excited oscillations by Theorem 5 .

Theorem 15. Let $\tau \in(0, \pi / \omega)$ and (33) hold. Equation (34) is oscillatory in the next two cases:

(i) $q=p$ and $\lambda$ is large enough;

(ii) $q>p, \lambda>0, \rho>0$, and at least one of $\lambda$ and $\rho$ is large enough.

Proof. Let $r(t) \equiv 1, A(v)=|v|^{p-1}, F(t, u)=\Phi(t, u), G(t, u) \equiv$ $0, e(t)=\cos (\omega t)$, and

$$
B(t, u, v)=\omega_{0}^{2} u+\frac{\mu_{1}|u|^{r_{1}} \operatorname{sgn}(u)}{\left(\mu_{2}+\mu_{3} u^{2}\right)^{r_{2}}}+\sum_{i=1}^{m} \beta_{i}(t)|u|^{\alpha_{i}-1} u .
$$

It is easy to check that all assumptions of Theorem 5 are fulfilled with respect to the sequence $a_{n}=-\pi / 2 \omega+n \pi / \omega+\tau$ and $b_{n}=\pi / 2 \omega+n \pi / \omega+\tau$, where $a_{n}<b_{n}$ since it is supposed that $\tau<\pi / \omega$. Hence, Theorem 5 proves this theorem.

Remark 16. Even in the linear forced case $(e(t) \not \equiv 0)$, it is not easy to establish the oscillations of all solutions, since the oscillation and nonoscillation can occur simultaneously. The most simple and important example for the coincidence of oscillation and nonoscillation is the following linear forced differential equation: $x^{\prime \prime}+(2 / t) x^{\prime}+x=2 / t, t>0$, that allows an oscillatory solution $x_{1}(t)=(3 \sin t) / t+2 / t$ and a nonoscillatory solution $x_{2}(t)=2 / t$. This is not possible in the linear case with $e(t) \equiv 0$, because of Sturm's separation theorem.

\section{Parametrically Excited Oscillations and Well-Known Oscillation Criteria}

In this section, we would like to draw the reader's attention to the fact that the parametrically excited oscillations have 
been already appearing in some published papers on the oscillation of functional differential equations, but only in some examples illustrating certain main oscillation criteria. However, with the help of our main results in which the parametrically excited oscillations are studied in a general setting, the equations from these examples are replaced with general ones also having parameters $\lambda$ and $\mu$.

In [1] (see also [2, Example 3.1] with $\tau=0$, [3, Example 3.1], and [4, Section 3]), the author considers the oscillation of the second-order delay differential equation:

$$
x^{\prime \prime}(t)+f(t)|x(\tau(t))|^{\gamma} \operatorname{sgn} x(\tau(t))=e(t),
$$

in the linear case $(\gamma=1)$ and the superlinear $(\gamma>1)$. In the linear case (analogously for the superlinear case see [1, Theorem 2]), the author proved the following oscillation criterion. In what follows, we denote

$$
D(a, b)=\left\{u \in C^{1}([a, b], \mathbb{R}): u(t) \not \equiv 0, u(a)=u(b)=0\right\} \text {. }
$$

Theorem 17 ([1, Theorem 1]). Suppose that, for any $T \geq 0$, there exist constants $a_{1}, b_{1}, a_{2}, b_{2}$ such that $T \leq a_{1}<b_{1}, T \leq$ $a_{2}<b_{2}$, and $f(t) \geq 0$ on $\left[\tau\left(a_{1}\right), b_{1}\right] \cup\left[\tau\left(a_{2}\right), b_{2}\right], e(t) \leq 0$ on $\left[\tau\left(a_{1}\right), b_{1}\right]$, and $e(t) \geq 0$ on $\left[\tau\left(a_{2}\right), b_{2}\right]$. If there exists $u \in$ $D\left(a_{i}, b_{i}\right), i=1,2$, such that

$$
\int_{a_{i}}^{b_{i}}\left[u^{2}(t) f(t) \frac{\tau(t)-\tau\left(a_{i}\right)}{t-\tau\left(a_{i}\right)}-\left(u^{\prime}(t)\right)^{2}\right] d t \geq 0,
$$

then (36) with $\gamma=1$ is oscillatory.

Previous criterion has been applied on the following particular equation:

$$
\begin{aligned}
& x^{\prime \prime}(t)+\lambda \sin (t)\left|x\left(t-\frac{\pi}{4}\right)\right|^{\gamma} \\
& \quad \times \operatorname{sgn} x\left(t-\frac{\pi}{4}\right)=\cos (t), \quad t \geq 0,
\end{aligned}
$$

where $\lambda \geq 0$ and $\gamma=1$. Applying Theorem 17 to (39), the author proved that (39) is oscillatory provided the following inequality:

$$
\lambda \int_{a_{i}}^{b_{i}} \sin ^{2}(2 t) \cos ^{2}(2 t) \sin (t) \frac{t-a_{i}}{t-a_{i}+\pi / 4} d t \geq \frac{\pi}{2},
$$

holds for sufficiently large $\lambda$. Thus, the oscillation of (39) is excited by the large enough parameter $\lambda$. However, according to Theorems 5 and 6 , we are able to show that the next parametric equation that corresponds to general equation (36)

$$
x^{\prime \prime}(t)+\lambda f(t)|x(\tau(t))|^{\gamma} \operatorname{sgn} x(\tau(t))=e(t)
$$

is oscillatory provided $\lambda$ is large enough, where $p_{1}=p_{2}=\gamma$, $\mu=0$, and $\rho=1$.

Next, in [5] (see also [6-8]), the authors consider the oscillation of the following class of second-order differential equations with delay and advanced arguments:

$$
\begin{aligned}
& \left(r(t) x^{\prime}(t)\right)^{\prime}+f(t)|x(\tau(t))|^{p_{1}} \operatorname{sgn} x(\tau(t)) \\
& \quad+g(t)|x(\sigma(t))|^{p_{2}} \operatorname{sgn} x(\sigma(t))=e(t), \quad t \geq 0,
\end{aligned}
$$

where $p_{1}, p_{2} \geq 1$. When $p_{1}=p_{2}=1$ the authors prove the following result (for other cases see [5, Theorems 3.2, 3.3, and 3.4].

Theorem 18 ([5, Theorem 3.1]). Suppose that, for any $T \geq$ 0 , there exist intervals $\left[\tau\left(a_{1}\right), b_{1}\right],\left[\tau\left(a_{2}\right), b_{2}\right],\left[c_{1}, \sigma\left(d_{1}\right)\right]$, and $\left[c_{2}, \sigma\left(d_{2}\right)\right]$ contained in $[T, \infty)$ such that $a_{1}<b_{1}, a_{2}<b_{2}$, $c_{1}<d_{1}, c_{2}<d_{2}$, and

$$
\begin{aligned}
& f(t) \geq 0 \quad \text { on }\left[\tau\left(a_{1}\right), b_{1}\right] \cup\left[\tau\left(a_{2}\right), b_{2}\right], \\
& g(t) \geq 0 \quad \text { on }\left[c_{1}, \sigma\left(d_{1}\right)\right] \cup\left[c_{2}, \sigma\left(d_{2}\right)\right], \\
& e(t) \leq 0 \quad \text { on }\left[\tau\left(a_{1}\right), b_{1}\right] \cup\left[c_{1}, \sigma\left(d_{1}\right)\right], \\
& e(t) \geq 0 \quad \text { on }\left[\tau\left(a_{2}\right), b_{2}\right] \cup\left[c_{2}, \sigma\left(d_{2}\right)\right],
\end{aligned}
$$

and $c_{i}=\tau\left(a_{i}\right), d_{i}=a_{i}$, and $b_{i}=\sigma\left(d_{i}\right), i=1$, 2. If there exist $u_{1} \in D\left(a_{i}, b_{i}\right)$ and $u_{2} \in D\left(c_{i}, d_{i}\right)$ such that either

$$
\int_{a_{i}}^{b_{i}}\left[u_{1}^{2}(t) f(t) \frac{\tau(t)-\tau\left(a_{i}\right)}{t-\tau\left(a_{i}\right)}-\left(u_{1}^{\prime}(t)\right)^{2} r(t)\right] d t \geq 0
$$

or

$$
\int_{c_{i}}^{d_{i}}\left[u_{2}^{2}(t) f(t) \frac{\sigma\left(d_{i}\right)-\sigma(t)}{\sigma\left(d_{i}\right)-t}-\left(u_{2}^{\prime}(t)\right)^{2} r(t)\right] d t \geq 0,
$$

for $i=1,2$, then (42) with $p_{1}=p_{2}=1$ is oscillatory.

As a consequence of this result, it has been concluded that the particular equation

$$
\begin{aligned}
& \left(r(t) x^{\prime}(t)\right)^{\prime}+\lambda \sin (t) x\left(t-\frac{\pi}{12}\right) \\
& \quad+\mu \cos (t) x\left(t+\frac{\pi}{6}\right)=\cos (2 t), \quad t \geq 0,
\end{aligned}
$$

is oscillatory provided either $\lambda$ or $\mu$ is large enough. However, by following Theorems 5 and 6 , one can obtain the same conclusion for the following general equation associated with (42):

$$
\begin{gathered}
\left(r(t) x^{\prime}(t)\right)^{\prime}+\lambda f(t)|x(\tau(t))|^{p_{1}} \operatorname{sgn} x(\tau(t)) \\
+\mu g(t)|x(\sigma(t))|^{p_{2}} \operatorname{sgn} x(\sigma(t))=e(t) .
\end{gathered}
$$

Related observation can be done with [8, Example 3.3] and [9, Example 2.1], where the quasilinear second-order functional differential equations have been considered. It is left to the reader.

\section{Some Open Questions and Comments}

In this section, we discuss some problems related to our main results that are not studied here.

(1) Quasiperiodic Case. In the theory of nonlinear oscillators, a particularly important case occurs when the periodic coefficients in the oscillator do not have any common period. It is called the quasiperiodic (or two-frequency) nonlinear 
oscillator and studied, for instance, in [50-52]. Since in Theorems 5, 6, and 7 we assume that the corresponding periodic functions have a common period, it is natural to pose the next question.

Open Question 1. Is it possible to derive sufficient conditions for the oscillation of (27) in the case when $f(t)$ and $g(t)$ (resp., $f(t), g(t)$, and $h(t))$ are two (resp., three) periodic functions not having a common period?

(2) Equation with More Functional Arguments. Next, regarding some second-order functional differential equations considered in the references of this paper, more than two nonlinear functional terms are appearing and, therefore, instead of main equation (1) and corresponding particular equation (27) considered in Theorems 5, 6, and 7, we suggest the following classes of equations:

$$
\begin{aligned}
& \left(r(t) A\left(x^{\prime}(t)\right)\right)^{\prime}+B\left(t, x(t), x^{\prime}(t)\right) \\
& +\sum_{k=1}^{m_{1}} \lambda_{k} F_{k}\left(t, x\left(\tau_{k}(t)\right)\right) \\
& \quad+\sum_{k=1}^{m_{2}} \mu_{k} G_{k}\left(t, x\left(\sigma_{k}(t)\right)\right)=\rho e(t),
\end{aligned}
$$

where $0 \leq \tau_{k}(t) \leq t, \lim _{t \rightarrow \infty} \tau_{k}(t)=\infty, \sigma_{k}(t) \geq t, m_{1}, m_{2} \in$ $\mathbb{N}$, and

$$
\begin{aligned}
& \left(r(t) A\left(x^{\prime}(t)\right)\right)^{\prime}+B\left(t, x(t), x^{\prime}(t)\right) \\
& \quad+\sum_{k=1}^{m_{1}} \lambda_{k} f_{k}(t)\left|x\left(t-\tau_{k}\right)\right|^{p_{k}} \operatorname{sgn}\left(x\left(t-\tau_{k}\right)\right) \\
& \quad+\sum_{k=1}^{m_{2}} \mu_{k} g_{k}(t)\left|x\left(t+\sigma_{k}\right)\right|^{q_{k}} \operatorname{sgn}\left(x\left(t+\sigma_{k}\right)\right)=\rho e(t),
\end{aligned}
$$

where $\lambda_{k}, \mu_{k}, \rho, \tau_{k}, \sigma_{k} \geq 0$ and $p_{k}, q_{k}>0$.

Comment. We suggest the reader to enlarge the main results of this paper to (48) and (49).

(3) Damped Duffing Equation. In the application, the Duffing equation (34) is often appearing with the linear damped term $x^{\prime}(t)$; that is,

$$
x^{\prime \prime}+d_{0} x^{\prime}+\omega_{0}^{2} x+\beta x^{3}+\lambda \Phi(x(t-\tau))=\rho \cos (\omega t),
$$

where $d_{0}$ is the damped coefficient which can, in an active way, influence various behaviours of (50). Since $B\left(t, x(t), x^{\prime}(t)\right)=d_{0} x^{\prime}(t)$ does not satisfy the required assumption (4), we are not able to apply our main results to (50). Hence we pose the following question.

Open Question 2. Is it possible to obtain the parametrically excited oscillation for (1) in the case when the damped term $B(t, u, v)$ satisfies a larger condition than (4) in which the linear damped term $\beta x^{\prime}(t)$ is especially included?

(4) Functional Argument in Damped Term. In a class of Duffing equations, we have two time delayed feedback and, hence, besides the control gain parameter $\lambda_{1}$ another parameter $\lambda_{2}$ appears, the so-called velocity gain parameter. Hence, instead of (34) one can consider

$$
\begin{aligned}
x^{\prime \prime} & +d_{0} x^{\prime}+\omega_{0}^{2} x+\beta x^{3}+\lambda_{1} x(t-\tau) \\
& +\lambda_{2} x^{\prime}(t-\tau)=\rho \cos (\omega t) .
\end{aligned}
$$

Therefore, we suggest the following problem for further study.

Open Question 3. Is it possible to obtain the parametrically excited oscillation for the following more general functional differential equation than (1) in which the functional argument appears in the damped term too, as follows:

$$
\begin{aligned}
\left(r(t) A\left(x^{\prime}(t)\right)\right)^{\prime}+B\left(t, x(t), x^{\prime}(t)\right)+\lambda_{1} F(t, x(\tau(t))) \\
+\lambda_{2} H\left(t, x^{\prime}(\tau(t))\right)=\rho e(t), \quad t \geq t_{0},
\end{aligned}
$$

or

$$
\begin{gathered}
\left(r(t) A\left(x^{\prime}(t)\right)\right)^{\prime}+B\left(t, x(t), x^{\prime}(t)\right)+\mu_{1} G(t, x(\sigma(t))) \\
+\mu_{2} H\left(t, x^{\prime}(\sigma(t))\right)=\rho e(t), \quad t \geq t_{0} ?
\end{gathered}
$$

About known oscillation criteria for the second-order functional differential equations having the functional argument in the damped term, we refer the reader to, for instance, [53] and the references therein.

\section{Proofs of Main Results}

The proof of Lemma 1 is based on the following three steps: two working forms of condition (6) (see Lemmas 19 and 20), the existence of an explosive solution of a suitable Riccati differential inequality (see Proposition 22), and a comparison principle (see Proposition 24).

Lemma 19 (a necessary condition to (6)). Let $0<r(t) \leq r_{0}$ on $\left[t_{0}, \infty\right)$. If assumption (6) is fulfilled, then there is a positive real number $\varepsilon$ such that

$$
\frac{1}{\pi_{*}} \int_{J} \min \left\{\frac{p}{(\varepsilon r(t))^{\gamma-1}}, \varepsilon k(\lambda, \mu, \rho) Q(t)\right\} d t \geq 1,
$$

for all $\lambda \geq \lambda_{0}, \mu \geq \mu_{0}$, and $\rho \geq \rho_{0}$ and some $\left(\lambda_{0}, \mu_{0}, \rho_{0}\right) \in \mathbb{R}_{+}^{3}$.

Proof. Since $0<r(t) \leq r_{0}$ for $t \geq t_{0}$, we conclude that, for

$$
\varepsilon=\left(\frac{p}{r_{0}^{\gamma-1} k(\lambda, \mu, \rho) \max _{t \in \bar{J}} Q(t)}\right)^{1 / \gamma}, \quad(\lambda, \mu, \rho) \in \mathbb{R}_{+}^{3},
$$

it holds that $p /(\varepsilon r(t))^{\gamma-1} \geq p /\left(\varepsilon r_{0}\right)^{\gamma-1}=\varepsilon k(\lambda, \mu, \rho)$ $\max _{t \in \bar{J}} Q(t) \geq \varepsilon k(\lambda, \mu, \rho) Q(t), t \in J$, and hence

$$
\begin{gathered}
\int_{J} \min \left\{\frac{p}{(\varepsilon r(t))^{\gamma-1}}, \varepsilon k(\lambda, \mu, \rho) Q(t)\right\} d t \\
=\varepsilon k(\lambda, \mu, \rho) \int_{J} Q(t) d t .
\end{gathered}
$$


On the other hand, from (6) we observe

$$
\frac{1}{\pi_{*}} \int_{J} Q(t) d t \geq \frac{r_{0}^{1-(1 / \gamma)}}{p^{1 / \gamma}[k(\lambda, \mu, \rho)]^{1-(1 / \gamma)}}\left(\max _{t \in \bar{J}} Q(t)\right)^{1 / \gamma}
$$

which, together with (55) and (56), gives

$$
\begin{aligned}
& \frac{1}{\pi_{*}} \int_{J} \min \left\{\frac{p}{(\varepsilon r(t))^{\gamma-1}}, \varepsilon k(\lambda, \mu, \rho) Q(t)\right\} d t \\
& \quad=\varepsilon k(\lambda, \mu, \rho) \frac{1}{\pi_{*}} \int_{J} Q(t) d t \\
& \geq \varepsilon \frac{r_{0}^{1-(1 / \gamma)}}{p^{1 / \gamma}}[k(\lambda, \mu, \rho)]^{1 / \gamma}\left(\max _{t \in \bar{J}} Q(t)\right)^{1 / \gamma}=1,
\end{aligned}
$$

for all $n \geq n_{0}, \lambda \geq \lambda_{0}, \mu \geq \mu_{0}$, and $\rho \geq \rho_{0}$. It proves this lemma.

Lemma 20 (an equivalent condition to (54)). Assumption (54) is fulfilled if and only if there is a real number $\varepsilon>0$ and a continuous function $K(t) \geq 0, t \in \bar{J}$, such that

$$
\begin{aligned}
c_{0}:= & \int_{J} K(t) d t>0, \quad \frac{K(t)}{c_{0}} \leq \frac{1}{\pi_{*}} \\
& \times \min \left\{\frac{p}{(\varepsilon r(t))^{\gamma-1}}, \varepsilon k(\lambda, \mu, \rho) Q(t)\right\},
\end{aligned}
$$

for allt $\in J, \lambda \geq \lambda_{0}, \mu \geq \mu_{0}$, and $\rho \geq \rho_{0}$ and some $\left(\lambda_{0}, \mu_{0}, \rho_{0}\right) \in$ $\mathbb{R}_{+}^{3}$.

Proof. This proof is very elementary. Indeed, if (54) holds, then the function $K(t)$ and number $c_{0}$, defined by

$$
\begin{aligned}
K(t) & =\frac{1}{\pi_{*}} \min \left\{\frac{p}{(\varepsilon r(t))^{\gamma-1}}, \varepsilon k(\lambda, \mu, \rho) Q(t)\right\}, \\
c_{0} & :=\int_{J} K(t) d t,
\end{aligned}
$$

obviously satisfy $c_{0} \geq 1$ and $K(t) / c_{0} \leq K(t)=\left(1 / \pi_{*}\right)$ $\min \left\{p /(\varepsilon r(t))^{\gamma-1}, \varepsilon k(\lambda, \mu, \rho) Q(t)\right\}$ which shows (59). Conversely, if (59) holds, then, integrating both sides of the second inequality in (59), we obtain

$$
\int_{J} \frac{1}{\pi_{*}} \min \left\{\frac{p}{(\varepsilon r(t))^{\gamma-1}}, \varepsilon k(\lambda, \mu, \rho) Q(t)\right\} \geq \int_{J} \frac{K(t)}{c_{0}} d t=1,
$$

which shows (54).

In conclusion, according to previous two lemmas, we see that supposed condition (6) implies (59), which plays an important role in the proof of the main results.

The second step in the proof of Lemma 1 is to prove the existence of a function $\omega(t)$ which blows up in the finite time and satisfies a generalized Riccati differential lower inequality; we briefly present the existence and properties of the so-called generalized tangent type function. In what follows, let $\pi_{*}$ be a positive real number defined in (3). Let us remark that $\alpha(s)=s^{\gamma}, \gamma>1$, implies $\pi_{*}=(2 \pi) /(\gamma \sin (\pi / \gamma))$, see, for instance, [54], and obviously for $\gamma=2$ we have $\pi_{*}=\pi$.

Lemma 21. Let $\alpha:[0, \infty) \rightarrow[0, \infty)$ be a continuous function such that

$$
\int_{0}^{\infty} \frac{d \tau}{1+\alpha(\tau)}<\infty
$$

Then there is a real number $\pi_{*}>0$ and a function $z=z(s)$, $z \in C^{1}\left(\left(-\pi_{*} / 2, \pi_{*} / 2\right), \mathbb{R}\right)$, such that

$$
\begin{aligned}
\frac{d z}{d s} & =1+\alpha(|z(s)|), \quad s \in\left(-\frac{\pi_{*}}{2}, \frac{\pi_{*}}{2}\right), \\
z(0) & =0 .
\end{aligned}
$$

Moreover, $z(s)$ is increasing and odd:

$$
\begin{array}{r}
\lim _{s \rightarrow \pi_{*} / 2} z(s)=\infty, \quad \pi_{*}=\frac{2 \pi}{\gamma \sin (\pi / \gamma)} \quad \text { for } \alpha(s)=s^{\gamma}, \\
\gamma>1 .
\end{array}
$$

In particular, for $\alpha(s)=s^{2}$, one can take $z(s)=\tan (s)$ and $\pi_{*}=\pi$.

Proof. Let $Z=Z(t), t \in \mathbb{R}$, be a function defined by

$$
Z(t)=\int_{0}^{t} \frac{1}{1+\alpha(|\tau|)} d \tau, \quad t \in \mathbb{R}
$$

The function $Z(t)$ is well defined since $\alpha(s)$ is positive and continuous on $[0, \infty), Z(t)$ is increasing and odd function, and

$$
\begin{gathered}
\frac{d Z}{d t}=\frac{1}{1+\alpha(|t|)}, \quad t \in \mathbb{R}, \\
Z(0)=0, \quad Z \in C^{1}(\mathbb{R}, \mathbb{R}) .
\end{gathered}
$$

Moreover, because of (62), there is a real number $\pi_{*}>0$ such that

$$
\frac{\pi_{*}}{2}=\int_{0}^{\infty} \frac{d \tau}{1+\alpha(\tau)} .
$$

Thus, $Z: \mathbb{R} \rightarrow\left(-\pi_{*} / 2, \pi_{*} / 2\right)$ and there exists an inverse function $Z^{-1}=Z^{-1}(s)$ of the original function $Z=Z(t)$ and $Z^{-1}:\left(-\pi_{*} / 2, \pi_{*} / 2\right) \rightarrow \mathbb{R}$. Also, from $Z\left(Z^{-1}(s)\right)=s$ and $d Z / d t \neq 0$ on $\mathbb{R}$, we also derive that $d Z^{-1} / d s \neq 0$ on its domain $\left(-\pi_{*} / 2, \pi_{*} / 2\right)$ and

$$
\frac{d Z}{d t}\left(Z^{-1}(s)\right)=\frac{1}{\left(d Z^{-1} / d s\right)}, \quad s \in\left(-\frac{\pi_{*}}{2}, \frac{\pi_{*}}{2}\right) .
$$

Putting $t=Z^{-1}(s)$ for $s \in\left(-\pi_{*} / 2, \pi_{*} / 2\right)$ into (66) and using (68) we easily obtain

$$
\begin{aligned}
\frac{d Z^{-1}}{d s} & =1+\alpha\left(\left|Z^{-1}(s)\right|\right), \quad s \in\left(-\frac{\pi_{*}}{2}, \frac{\pi_{*}}{2}\right), \\
Z^{-1}(0) & =0, \quad Z^{-1} \in C^{1}\left(\left(-\frac{\pi_{*}}{2}, \frac{\pi_{*}}{2}\right), \mathbb{R}\right) .
\end{aligned}
$$


Moreover, from (67) we have $\lim _{s \rightarrow \pi_{*} / 2} Z^{-1}(s)=Z^{-1}$ $\left(\lim _{t \rightarrow \infty} Z(t)\right)=\lim _{t \rightarrow \infty} Z^{-1} Z(t)=\lim _{t \rightarrow \infty} t=\infty$. Thus, if we set $z(s):=Z^{-1}(s)$, then previous two statements and (67) prove this lemma.

Next, we prove the main result of this section.

Proposition 22. Let (2) and (6) hold, where $J=(a, b)$. Let $\varepsilon>$ 0 be a real number and let $K(t) \geq 0, t \in[a, b]$, be a continuous function, both obtained in Lemma 20. Let $\pi_{*}$ be from (3) and $c_{0}$ from (59), and let $R_{a} \in \mathbb{R}$ be an arbitrary real number. If $z=z(s)$ is the generalized tangens function defined in (63) and $V(t)$ is a function defined by

$$
V(t)=\frac{\pi_{*}}{c_{0}} \int_{a}^{t} K(\tau) d \tau+z^{-1}\left(R_{a}\right), \quad t \in[a, b],
$$

then there is a $T_{a}^{*} \in[a, b)$ such that

$$
V\left(T_{a}^{*}\right)=\frac{\pi_{*}}{2}, \quad V\left(\left[a, T_{a}^{*}\right)\right) \subset\left(-\frac{\pi_{*}}{2}, \frac{\pi_{*}}{2}\right) .
$$

Moreover, for a function $\underline{\omega}(t)$ defined by

$$
\underline{\omega}(t)=z(V(t)), \quad t \in\left[a, T_{a}^{*}\right),
$$

one has $\underline{\omega}(a)=R_{a}, \lim _{t \rightarrow T_{a}^{*}} \underline{\omega}(t)=\infty$, and

$$
\begin{aligned}
\frac{d \underline{\omega}}{d t} \leq & \frac{p}{(\varepsilon r(t))^{\gamma-1}} \alpha(|\underline{\omega}(t)|) \\
& +\varepsilon k(\lambda, \mu, \rho) Q(t), \quad t \in\left(a, T_{a}^{*}\right),
\end{aligned}
$$

where the numbers $p$ and $\gamma$ are from (3) and the functions $k(\lambda, \mu, \rho)$ and $Q(t)$ are from $(6)$.

Proof. Under assumptions (2) and (6) and because of Lemmas 19 and 20, we obtain $\varepsilon>0$ and $K(t)>0, t \in[a, b]$, satisfying inequality (59).

Next, since $z^{-1}\left(R_{a}\right) \in\left(-\pi_{*} / 2, \pi_{*} / 2\right)$ (see Lemma 21$)$, from (70) we directly obtain

$$
V(a)=z^{-1}\left(R_{a}\right)<\frac{\pi_{*}}{2}, \quad V(b)=\pi_{*}+z^{-1}\left(R_{a}\right)>\frac{\pi_{*}}{2} .
$$

Since $K \in C([a, b],[0, \infty))$ we obtain $V \in C([a, b], \mathbb{R}) \cap$ $C^{1}((a, b), \mathbb{R})$, and from (74) we observe that there exist numbers $T_{a}^{*} \in(a, b)$ such that $V\left(T_{a}^{*}\right)=\pi_{*} / 2$. Also, $K(t) / c_{0} \geq$ 0 gives $V\left(\left[a, T_{a}^{*}\right)\right) \subset\left(-\pi_{*} / 2, \pi_{*} / 2\right)$, which proves statement (71). Moreover, it together with Lemma 21 and (72) proves that

$$
\lim _{t \rightarrow T_{a}^{*}} \underline{\omega}(t)=\lim _{t \rightarrow T_{a}^{*}} z(V(t))=z\left(\frac{\pi_{*}}{2}\right)=\infty .
$$

Next, according to (59), (63), and (72), we make the following calculation on the interval $\left[a, T_{a}^{*}\right)$ :

$$
\begin{aligned}
\underline{\omega}^{\prime}(t) & =z^{\prime}(V(t)) V^{\prime}(t)=[1+\alpha(|z(V(t))|)] \frac{\pi_{*}}{c_{0}} K(t) \\
& =[1+\alpha(|\underline{\omega}(t)|)] \frac{\pi_{*}}{c_{0}} K(t) \\
& \leq \frac{p}{(\varepsilon r(t))^{\gamma-1}} \alpha(|\underline{\omega}(t)|)+\varepsilon k(\lambda, \mu, \rho) Q(t) .
\end{aligned}
$$

Thus, all assertions of this proposition are proved.
Remark 23. In the proof of the main result, the number $R_{a}$ is determined by $R_{a}=\bar{\omega}(a)$, where $\bar{\omega}(t)$ denotes a function associated with a nonoscillatory solution and it is given by (84) below.

The third step in the proof of Lemma 1 is to show the following pointwise comparison principle for the functions $\underline{\omega}$ and $\bar{\omega}$ satisfying, respectively, the lower and upper differential inequalities (73) and

$$
\frac{d \bar{\omega}}{d t} \geq \frac{p}{(\varepsilon r(t))^{\gamma-1}} \alpha(|\bar{\omega}(t)|)+\varepsilon k(\lambda, \mu, \rho) Q(t), \quad t \in(a, b) .
$$

Proposition 24. Let $[a, b) \subset\left[t_{0}, \infty\right)$ be an arbitrary interval. One supposes that all coefficients of Riccati differential inequalities (73) and (77) are continuous and strictly positive functions. Let $\underline{\omega}, \bar{\omega} \in C^{1}([a, b), \mathbb{R})$ be two functions satisfying, respectively, (73) and (77) on the interval $[a, b)$. Then

$$
\underline{\omega}(a) \leq \bar{\omega}(a) \text { implies } \underline{\omega}(t) \leq \bar{\omega}(t) \quad \forall t \in[a, b) .
$$

Proof. Let $H(t, u)$ be a function defined by

$$
\begin{array}{r}
H(t, u)=\frac{p}{(\varepsilon r(t))^{\gamma-1}} \alpha(|u|)+\varepsilon k(\lambda, \mu, \rho) Q(t), \\
t \in[a, b), u \in \mathbb{R} .
\end{array}
$$

Let $I \subset[a, b)$ and $M>0$ be arbitrary. For any two $u_{1}$, $u_{2},-M \leq u_{1}<u_{2} \leq M$, let $I_{12}$ be an interval defined by $I_{12}=\left(\min \left\{\left|u_{1}\right|,\left|u_{2}\right|\right\}, \max \left\{\left|u_{1}\right|,\left|u_{2}\right|\right\}\right)$. Since $\alpha(s)$ is a $C^{1}$ function on $[0, \infty)$, we know by the Lagrange mean value theorem applied on $I_{12}$ that there is a $\xi \in I_{12}$ such that

$$
\begin{aligned}
\frac{\alpha\left(\left|u_{2}\right|\right)-\alpha\left(\left|u_{1}\right|\right)}{u_{2}-u_{1}} & \leq \frac{\left|\alpha\left(\left|u_{2}\right|\right)-\alpha\left(\left|u_{1}\right|\right)\right|}{u_{2}-u_{1}} \\
& =\left|\alpha^{\prime}(\xi)\right| \frac{|| u_{2}|-| u_{1}||}{u_{2}-u_{1}} \leq\left|\alpha^{\prime}(\xi)\right| \\
& \leq \max _{s \in I_{12}}\left|\alpha^{\prime}(s)\right|
\end{aligned}
$$

since ||$u_{2}|-| u_{1}|| \leq u_{2}-u_{1}$. Hence for any $t \in I$ and $u_{1}, u_{2}$, $-M \leq u_{1}<u_{2} \leq M$, we have

$$
\begin{aligned}
\frac{H\left(t, u_{2}\right)-H\left(t, u_{1}\right)}{u_{2}-u_{1}} & =\rho_{0}(t) \frac{\alpha\left(\left|u_{2}\right|\right)-\alpha\left(\left|u_{1}\right|\right)}{u_{2}-u_{1}} \\
& \leq \rho_{0}(t) \max _{s \in I_{12}}\left|\alpha^{\prime}(s)\right|:=L_{0}(t) .
\end{aligned}
$$

Thus, the function $H(t, u)$ from (79) satisfies required condition of [55, Lemma 19] and, applying it to (73) and (77), we prove this proposition.

Proof of Lemma 1. On the contrary, let $x(t)$ be a solution of (1) such that

$$
x(t) \neq 0 \text { on }(\tau(\tau(a)), \sigma(\sigma(d)))
$$


that is, $x(t)>0$ on $(\tau(\tau(a)), \sigma(\sigma(d)))$ or $x(t)<0$ on $(\tau(\tau(a)), \sigma(\sigma(d)))$ since $x(t)$ is a continuous function on $\left[t_{0}, \infty\right)$. Let, for instance,

$$
x(t)>0 \quad \text { on }(\tau(\tau(a)), \sigma(\sigma(d))) .
$$

Another case can be analogously treated; let us see the comment at the end of this proof. In particular from (83) we have $x(t)>0$ on $(\tau(\tau(a)), \sigma(\sigma(b)))$ which implies (since $\tau(t)$ and $\sigma(t)$ are increasing functions) $x(s)>0$ for all $s \in$ $(\tau(a), \sigma(b)) \cup(\tau(\tau(a)), \tau(\sigma(b))) \cup(\sigma(\tau(a)), \sigma(\sigma(b)))$, which yields $x(t)>0, x(\tau(t))>0$, and $x(\sigma(t))>0$ on $(\tau(a), \sigma(b))$. Hence, by assumption (7), we may use inequality (5) on the interval $(a, b)$.

Firstly, we show that the following classic Riccati transformation of $x(t)$ :

$$
\bar{\omega}(t)=-\varepsilon \frac{r(t) A\left(x^{\prime}(t)\right)}{|x(t)|^{p-1} x(t)}, \quad t \in(a, b), \quad \varepsilon>0,
$$

satisfies upper Riccati differential inequality (77). Let us remark that from (1) we have in particular

$$
\begin{aligned}
-\left(r(t) A\left(x^{\prime}(t)\right)\right)^{\prime}= & B\left(t, x(t), x^{\prime}(t)\right)+\lambda F(t, x(\tau(t))) \\
& +\mu G(t, x(\sigma(t)))-\rho e(t), \quad t \geq t_{0} .
\end{aligned}
$$

Taking the first derivative on both sides of (84) and using assumptions (3), (4), and (5) as well as equality (85) and $\left(|x(t)|^{p-1} x(t)\right)^{\prime}=p|x(t)|^{p-1} x^{\prime}(t)$, we obtain

$$
\begin{aligned}
& \frac{d \bar{\omega}}{d t}= \varepsilon p r(t) \frac{A\left(x^{\prime}(t)\right) x^{\prime}(t)}{|x(t)|^{p+1}} \\
&-\varepsilon \frac{1}{|x(t)|^{p^{-1} x(t)}}\left(r(t) A\left(x^{\prime}(t)\right)\right)^{\prime} \\
&= \operatorname{ppr}(t) \frac{A\left(x^{\prime}(t)\right) x^{\prime}(t)}{|x(t)|^{p+1}}+\frac{\varepsilon}{|x(t)|^{p-1} x(t)} \\
& \times\left[\lambda B\left(t, x(t), x^{\prime}(t)\right)+F(t, x(\tau(t)))\right. \\
& \geq \varepsilon p r(t) \frac{A\left(x^{\prime}(t)\right) x^{\prime}(t)}{|x(t)|^{p+1}}+\frac{\varepsilon}{|x(t)|^{p-1} x(t)} \\
& \times[\lambda F(t, x(\tau(t)))+\mu G(t, x(\sigma(t)))-\rho e(t)] \\
& \geq \varepsilon p r(t) \alpha\left(\frac{\left|A\left(x^{\prime}(t)\right)\right|}{|x(t)|^{p}}\right)+\varepsilon k(\lambda, \mu, \rho) Q(t) \\
&= \varepsilon p r(t) \alpha\left(\frac{|\bar{\omega}(t)|}{\varepsilon r(t)}\right)+\varepsilon k(\lambda, \mu, \rho) Q(t) \\
&(\varepsilon r(t))^{\gamma-1} \alpha(|\bar{\omega}(t)|)+\varepsilon k(\lambda, \mu, \rho) Q(t), \quad t \in(a, b) .
\end{aligned}
$$

Thus, according to inequality (5), it is shown that if $x(t)$ is a solution of (1) which satisfies (83), then the function $\bar{\omega}(t)$ defined by (84) satisfies the Riccati differential inequality (77) and $\bar{\omega} \in C((a, b), \mathbb{R})$. On the other hand, let $R_{a}$ be a real number defined by $R_{a}=\bar{\omega}(a)$. According to (6) and Lemma 19 we obtain (54) which together with Lemma 20 ensures that we may use Proposition 22 for such chosen real number $R_{a}$. Hence, we obtain a function $\underline{\omega}(t)$ defined by (72) which satisfies the lower Riccati differential inequality (73) on $\left[a, T_{a}^{*}\right), T_{a}^{*} \in(a, b)$, such that $\underline{\omega}(a)=R_{a}$ and $\lim _{t \rightarrow T_{a}^{*}} \underline{\omega}(t)=$ $\infty$. Therefore, by $\underline{\omega}(a)=R_{a}=\bar{\omega}(a)$ and Proposition 24, we conclude that $\lim _{t \rightarrow T_{a}^{*}} \bar{\omega}(t)=\infty$ too, which is a contradiction with the above conclusion saying that $\bar{\omega} \in C((a, b), \mathbb{R})$. Thus, hypothesis (82) is not true and, consequently, Lemma 1 is shown.

For the analogous case $x(t)<0$ on $(\tau(\tau(a)), \sigma(\sigma(d)))$, we also have $x(t)<0$ on $(\tau(\tau(c)), \sigma(\sigma(d)))$ which implies (since $\tau(t)$ and $\sigma(t)$ are increasing functions)

$$
\begin{aligned}
x(s)<0 \quad \forall s \in(\tau(c), \sigma(d)) & \cup(\tau(\tau(c)), \tau(\sigma(d))) \\
& \cup(\sigma(\tau(c)), \sigma(\sigma(d))),
\end{aligned}
$$

which yields $x(t)<0, x(\tau(t))<0$, and $x(\sigma(t))<0$ on $(\tau(c), \sigma(d))$. Now we can repeat the preceding procedure but on interval $(c, d)$ and using (8) instead of $(a, b)$ and (7).

Proof of Lemma 2. From assumption (10), we obtain the existence of an $n_{0} \in \mathbb{N}$ such that

$$
\int_{a_{n}}^{b_{n}} Q_{n}(t) d t \geq \frac{c_{0}}{2}\left(\max _{t \in\left[a_{n}, b_{n}\right]} Q_{n}(t)\right)^{1 / \gamma}, \quad n \geq n_{0} ;
$$

that is,

$$
\frac{2}{c_{0}} \int_{a_{n}}^{b_{n}} Q_{n}(t) d t \geq\left(\max _{t \in\left[a_{n}, b_{n}\right]} Q_{n}(t)\right)^{1 / \gamma}, \quad n \geq n_{0} .
$$

Now from (9) and previous inequality we deduce that for large enough $\lambda, \mu, \rho$, and $n$

$$
\begin{aligned}
& \frac{p^{1 / \gamma}}{r_{0}^{1-1 / \gamma}} \frac{[k(\lambda, \mu, \rho)]^{1-1 / \gamma}}{\pi_{*}} \int_{a_{n}}^{b_{n}} Q_{n}(t) d t \\
& \quad \geq \frac{2}{c_{0}} \int_{a_{n}}^{b_{n}} Q_{n}(t) d t \geq\left(\max _{t \in\left[a_{n}, b_{n}\right]} Q_{n}(t)\right)^{1 / \gamma},
\end{aligned}
$$

which shows (6). Thus, all assumptions of Lemma 1 are fulfilled and, hence, Lemma 2 immediately follows from Lemma 1.

Proof of Lemma 3. Obviously assumption (11) is a particular case of assumption (9). Hence, this proof is very similar to the proof of Lemma 2 and so it is left to the reader.

Proof of Lemma 4. It is clear that from assumption (13) we obtain

$$
\frac{1}{\left(\max _{t \in\left[a_{n}, b_{n}\right]} Q_{n}(t)\right)^{1 / \gamma}} \int_{a_{n}}^{b_{n}} Q_{n}(t) d t \geq \frac{c_{1}}{C_{0}^{1 / \gamma}}>0, \quad \forall n \geq n_{0} .
$$


Thus, hypothesis (12) is fulfilled and, therefore, Lemma 3 proves this lemma.

Proof of Theorems 5, 6, and 7. This proof is based on Lemma 4. In order to simplify notation, in many places in this proof we set $\tau(t)=t-\tau$ and $\sigma(t)=t+\sigma$. Since assumptions (2), (3), and (4) have been already supposed in Theorems 5, 6, and 7, in order to prove these theorems by Lemma 4, we are going to show that the functions $k(\lambda, \mu, \rho)$ and $Q_{n}(t)$ explicitly given, respectively, in (18), (21), or (24) and (19), (22), or (25) satisfy required conditions (11) and (13), respectively, and that every solution $x(t)$ of (27) satisfies conditions (7) and (8) with respect to functions $k(\lambda, \mu, \rho)$ and $Q_{n}(t)$, where $a=a_{2 n-1}, b=b_{2 n-1}, c=a_{2 n}$, and $d=b_{2 n}$.

The proof that the function $k(\lambda, \mu, \rho)$ given in (18), (21), or (24) satisfies (11). Passing to the limit in (18), (21), or (24) it is very simple to show (11).

The proof that the function $Q_{n}(t)$ given in (19), (22), or (25) satisfies the first claim in (13). From (25) we immediately obtain

$$
\begin{aligned}
& \left|\tau_{n}(t)\right|=\left|\left(\frac{t-a_{n}}{t-a_{n}+\tau}\right)^{p}\right| \leq 1, \\
& \left|\sigma_{n}(t)\right|=\left|\left(\frac{b_{n}-t}{b_{n}-t+\sigma}\right)^{p}\right| \leq 1 \quad \forall n \in \mathbb{N} .
\end{aligned}
$$

Next, by assumptions of this corollary we can conclude that there are three positive constants $f_{0}, g_{0}, e_{0}$ such that $|f(t)| \leq$ $f_{0}$ and $|g(t)| \leq g_{0}$ on $\left[t_{0}, \infty\right)$ in cases (i) and (ii) and $|e(t)| \leq e_{0}$ on $\left[t_{0}, \infty\right)$ in cases (iii) and (iv). Putting previous inequalities into (19), (22), or (25), for all $n \in \mathbb{N}$ and $t \in$ $\left[t_{0}, \infty\right)$, it holds that

$$
\left|Q_{n}(t)\right| \leq\left\{\begin{array}{l}
\frac{q}{p}\left(\frac{p}{q-p}\right)^{(q / p)-1} e_{0}^{1-(p / q)} f_{0}^{p / q}, \\
\text { delay case with } q>p, \\
\frac{q}{p}\left(\frac{p}{q-p}\right)^{(q / p)-1} e_{0}^{1-(p / q)} g_{0}^{p / q}, \\
\frac{p_{1}}{p}\left(\frac{p d v a n c e d \text { case with } q>p,}{2\left(p_{1}-p\right)}\right)^{\left(p_{1} / p\right)-1} e_{0}^{1-\left(p / p_{1}\right)} f_{0}^{p / q}+\frac{p_{2}}{p} \\
\times\left(\frac{p}{2\left(p_{2}-p\right)}\right)^{\left(p_{2} / p\right)-1} e_{0}^{1-\left(p / p_{2}\right)} g_{0}^{p / q}, \\
\text { delay-advanced case (i), } \\
e_{0}^{\eta_{0}} f_{0}^{\eta_{1}} g_{0}^{\eta_{2}} \prod_{i=0}^{2} \eta_{i}^{-\eta_{i}}, \\
\text { delay-advanced case (ii), }
\end{array}\right.
$$

which shows the first claim in (13).
The proof that the function $Q_{n}(t)$ given in (19), (22), or (25) satisfies the second claim in (13). Without loss of generality, we prove this claim only in case (i), since for other cases the proof follows analogously. In this sense, let $Q_{n}(t)=f(t) \tau_{n}(t)$. Since $a_{2 n+1}-a_{2 n-1} \leq T_{*}, b_{2 n+1}-b_{2 n-1} \geq T_{*}, a_{2 n+2}-a_{2 n} \leq T_{*}$, and $b_{2 n+2}-b_{2 n} \geq T_{*}$, where $T_{*}>0$ is the period of the function $f(t)$, we have $a_{2 n-1} \leq a_{1}+(n-1) T_{*}$ and $b_{2 n-1} \geq b_{1}+(n-1) T_{*}$, $n \in \mathbb{N}$. Hence

$$
\begin{aligned}
& \int_{a_{2 n-1}}^{b_{2 n-1}} Q_{n}(t) d t \\
& \quad=\int_{a_{2 n-1}}^{b_{2 n-1}} f(t)\left(\frac{t-a_{2 n-1}}{t-a_{2 n-1}+\tau}\right)^{p} d t \\
& \quad \geq \int_{a_{1}+(n-1) T_{*}}^{b_{1}+(n-1) T_{*}} f(t)\left(\frac{t-a_{1}-(n-1) T_{*}}{t-a_{1}-(n-1) T_{*}+\tau}\right)^{p} d t \\
& =\int_{a_{1}}^{b_{1}} f\left(s+(n-1) T_{*}\right)\left(\frac{s-a_{1}}{s-a_{1}+\tau}\right)^{p} d s \\
& =\int_{a_{1}}^{b_{1}} f(s)\left(\frac{s-a_{1}}{s-a_{1}+\tau}\right)^{p} d s,
\end{aligned}
$$

which proves that the integral on the left hand side does not depend on $n \in \mathbb{N}$; that is, the second claim in (13) is shown on $\left[a_{2 n-1}, b_{2 n-1}\right]$. This claim follows in the same way on $\left[a_{2 n}, b_{2 n}\right]$. Thus, the second claim in (13) is proved on $\left[a_{n}, b_{n}\right]$.

Next, to the end of this proof, let $x(t)$ be a solution of (1). In particular, it implies that $\left(r(t) A\left(x^{\prime}(t)\right)\right)^{\prime}=$ $-B\left(t, x(t), x^{\prime}(t)\right)-\lambda F(t, x(\tau(t)))-\mu G(t, x(\sigma(t)))+\rho e(t)$. It together with assumptions (15), (16), (20), and (23) easily gives the next two statements:

$$
\begin{aligned}
& \text { if } x(t)>0, \quad x(\tau(t))>0, \quad x(\sigma(t))>0 \\
& \text { on }\left(\tau\left(a_{2 n-1}\right), \sigma\left(b_{2 n-1}\right)\right), \\
& \text { then } x(t) \text { satisfies } r(t) A\left(x^{\prime}(t)\right) \leq 0 \\
& \text { on }\left(\tau\left(a_{2 n-1}\right), \sigma\left(b_{2 n-1}\right)\right), \quad n \geq n_{0} ; \\
& \text { if } x(t)<0, \quad x(\tau(t))<0, \quad x(\sigma(t))<0 \\
& \text { on }\left(\tau\left(a_{2 n}\right), \sigma\left(b_{2 n}\right)\right), \\
& \text { then } x(t) \text { satisfies } r(t) A\left(x^{\prime}(t)\right) \geq 0 \\
& \text { on }\left(\tau\left(a_{2 n}\right), \sigma\left(b_{2 n}\right)\right), \quad n \geq n_{0} .
\end{aligned}
$$

Now we need the following lemma. 
Lemma 25. Let $\tau_{a b}(t)$ and $\sigma_{a b}(t)$ be defined by

$$
\begin{aligned}
\tau_{a b}(t) & =\left(\frac{\tau(t)-\tau(a)}{t-\tau(a)}\right)^{p}, \\
\sigma_{a b}(t) & =\left(\frac{\sigma(b)-\sigma(t)}{\sigma(b)-t}\right)^{p}, \quad t \in(a, b),
\end{aligned}
$$

and let $x \in C^{2}\left(\left[T_{0}, \infty\right), \mathbb{R}\right)$ be an arbitrary function. If $\left(r(t) A\left(x^{\prime}(t)\right) \leq 0\right.$ for all $t \in(\tau(a), \sigma(b))$ or $\left(r(t) A\left(x^{\prime}(t)\right) \geq 0\right.$ for all $t \in(\tau(a), \sigma(b))$, then

$$
\frac{x(\tau(t))}{x(t)} \geq\left(\tau_{a b}(t)\right)^{1 / p}, \quad \frac{x(\sigma(t))}{x(t)} \geq\left(\sigma_{a b}(t)\right)^{1 / p}, t \in(a, b) .
$$

Since $A(v)$ is supposed to be odd and increasing function, just before (3), and $r(t)$ satisfies (14), the proof of Lemma 25 in the first case, that is, $r(t) A\left(x^{\prime}(t)\right) \leq 0$ for all $t \in(\tau(a), \sigma(b))$, is the same as the proof of [9, Corollaries 17 and 18]. But in the second case, that is, $r(t) A\left(x^{\prime}(t)\right) \geq 0$ for all $t \in(\tau(a), \sigma(b))$, the proof is as follows: if previous inequality holds, then $r(t) A\left(-x^{\prime}(t)\right) \leq 0$ for all $t \in(\tau(a), \sigma(b))$ and, therefore, to the function $-x(t)$ one can apply the first case of this lemma and consequently one obtains

$$
\begin{aligned}
& \frac{x(\tau(t))}{x(t)}=\frac{-x(\tau(t))}{-x(t)} \geq\left(\tau_{a b}(t)\right)^{1 / p}, \\
& \frac{x(\sigma(t))}{x(t)}=\frac{-x(\sigma(t))}{-x(t)} \geq\left(\sigma_{a b}(t)\right)^{1 / p}, \quad t \in(a, b),
\end{aligned}
$$

which proves this lemma in the second case.

Now, combining statements (95), (96), and (98), one easily obtains

$$
\begin{aligned}
& \begin{array}{r}
\text { if } x(t)>0, \quad x(\tau(t))>0, \quad x(\sigma(t))>0 \\
\text { on }\left(\tau\left(a_{2 n-1}\right), \sigma\left(b_{2 n-1}\right)\right), \\
\text { then } x(t) \text { satisfies } \frac{x(\tau(t))}{x(t)} \geq\left(\tau_{n}(t)\right)^{1 / p} \\
\text { on }\left(a_{2 n-1}, b_{2 n-1}\right), n \geq n_{0} ; \\
\text { if } x(t)<0, \quad x(\tau(t))<0, \quad x(\sigma(t))<0 \\
\text { on }\left(\tau\left(a_{2 n}\right), \sigma\left(b_{2 n}\right)\right), \\
\text { then } x(t) \text { satisfies } \frac{x(\sigma(t))}{x(t)} \geq\left(\sigma_{n}(t)\right)^{1 / p} \\
\text { on }\left(a_{2 n}, b_{2 n}\right), n \geq n_{0},
\end{array}
\end{aligned}
$$

where $\tau_{n}(t)$ and $\sigma_{n}(t)$ are defined in (26).

The proof that $x(t)$ satisfies (7) and (8). In this proof, we frequently use assumptions (16), (20), and (23) and statements (100) and (101). Also, because of (15) and $F(t, u)=$ $f(t)|u|^{p_{1}} \operatorname{sgn}(u), G(t, u)=g(t)|u|^{p_{2}} \operatorname{sgn}(u)$, in both cases $(100)$ and (101), we can simultaneously use

$$
\begin{gathered}
-e(t)\left(|x(t)|^{p-1} x(t)\right)^{-1}=|e(t)||x(t)|^{-p} \geq 0 \quad \text { on } J_{n}, \\
F(t, x(\tau(t)))\left(|x(t)|^{p-1} x(t)\right)^{-1} \\
\geq f(t)|x(\tau(t))|^{p_{1}}|x(t)|^{-p} \geq 0 \text { on } J_{n}, \\
G(t, x(\sigma(t)))\left(|x(t)|^{p-1} x(t)\right)^{-1} \\
\geq g(t)|x(\sigma(t))|^{p_{2}}|x(t)|^{-p} \geq 0 \text { on } J_{n}, \\
|x(\tau(t))||x(t)|^{-1}=\frac{x(\tau(t))}{x(t)}, \\
|x(\sigma(t))||x(t)|^{-1}=\frac{x(\sigma(t))}{x(t)} \text { on } J_{n},
\end{gathered}
$$

where $J_{n}=\left(a_{2 n-1}, b_{2 n-1}\right)$ in the case of $(100)$ and $J_{n}=\left(a_{2 n}, b_{2 n}\right)$ in the case of (101).

(i) Delay or Advanced Case with $q=p$. Since $q=p$, we obtain

$$
\begin{aligned}
& {[\lambda F(t, x(\tau(t)))+\mu G(t, x(\sigma(t)))-\rho e(t)]} \\
& \quad \times\left(|x(t)|^{p-1} x(t)\right)^{-1} \\
& \geq\left[\lambda f(t)|x(\tau(t))|^{p}+\mu g(t)|x(\sigma(t))|^{p}\right. \\
& \quad+\rho|e(t)|]|x(t)|^{-p} \\
& \geq\left[\lambda f(t)|x(\tau(t))|^{p}+\mu g(t)|x(\sigma(t))|^{p}\right]|x(t)|^{-p} \\
& =\lambda f(t)\left(\frac{x(\tau(t))}{x(t)}\right)^{p}+\mu g(t)\left(\frac{x(\sigma(t))}{x(t)}\right)^{p} \\
& \geq \lambda f(t) \tau_{n}(t)+\mu g(t) \sigma_{n}(t), \quad t \in J_{n},
\end{aligned}
$$

where the functions $\tau_{n}(t)$ and $\sigma_{n}(t)$ are defined in (26).

(ii) Delay Case with $q>p$. In this part we use the next elementary inequality:

$$
X^{\gamma}+(\gamma-1) Y^{\gamma} \geq \gamma X Y^{\gamma-1}, \quad \gamma>1, X, Y \geq 0
$$

Since $q>p$ and using (104) especially for

$$
\begin{aligned}
& \gamma=\frac{q}{p}>1, \quad X=(\lambda f(t))^{p / q}|x(\tau(t))|^{p}, \\
& Y=\left(\frac{p}{q-p} \rho|e(t)|\right)^{p / q},
\end{aligned}
$$


for all $t \in J_{n}$ we obtain

$$
\begin{aligned}
& {[\lambda F(t, x(\tau(t)))+\mu G(t, x(\sigma(t)))-\rho e(t)] } \\
& \quad \times\left(|x(t)|^{p-1} x(t)\right)^{-1} \\
&=[\lambda F(t, x(\tau(t)))-\rho e(t)]\left(|x(t)|^{p-1} x(t)\right)^{-1} \\
& \geq {\left[\lambda f(t)|x(\tau(t))|^{p_{1}}+\rho|e(t)|\right]|x(t)|^{-p} } \\
&= {\left[X^{\gamma}+(\gamma-1) Y^{\gamma}\right]|x(t)|^{-p} } \\
& \geq \frac{q}{p}(\lambda f(t))^{p / q}|x(\tau(t))|^{p} \\
& \times\left(\frac{p}{q-p} \rho|e(t)|\right)^{(p / q)((q / p)-1)}|x(t)|^{-p} \\
&= \lambda^{p / q} \rho^{1-(p / q)} \frac{q}{p}\left(\frac{p}{q-p}\right)^{1-(p / q)}|e(t)|^{1-(p / q)} \\
& \times(f(t))^{p / q}\left(\frac{x(\tau(t))}{x(t)}\right)^{p} \\
& \geq k(\lambda, \mu, \rho) \frac{q}{p}\left(\frac{p}{q-p}\right)^{1-(p / q)}|e(t)|^{1-(p / q)} \\
& \times(f(t))^{p / q} \tau_{n}(t),
\end{aligned}
$$

(106)

where the function $k(\lambda, \mu, \rho)$ is from (18).

(iii) Advanced Case with $q>p$. Using the same line of arguments as in the proof of the previous case, for all $t \in J_{n}$ we obtain

$$
\begin{aligned}
& {[\lambda F(t, x(\tau(t)))+\mu G(t, x(\sigma(t)))-\rho e(t)]} \\
& \quad \times\left(|x(t)|^{p-1} x(t)\right)^{-1} \\
& =[\mu G(t, x(\sigma(t)))-\rho e(t)]\left(|x(t)|^{p-1} x(t)\right)^{-1} \\
& \geq k(\lambda, \mu, \rho) \frac{q}{p}\left(\frac{p}{q-p}\right)^{1-(p / q)}|e(t)|^{1-(p / q)} \\
& \quad \times(g(t))^{p / q} \sigma_{n}(t),
\end{aligned}
$$

where the function $k(\lambda, \mu, \rho)$ is from (21). (iv) Superlinear Delay-Advanced Case. Since $p_{1}, p_{2}>p$, for all $t \in J_{n}$ we obtain

$$
\begin{aligned}
& {[\lambda F(t, x(\tau(t)))+\mu G(t, x(\sigma(t)))-\rho e(t)] } \\
& \times\left(|x(t)|^{p-1} x(t)\right)^{-1} \\
&= {\left[\lambda F(t, x(\tau(t)))-\frac{\rho}{2} e(t)\right]\left(|x(t)|^{p-1} x(t)\right)^{-1} } \\
&+\left[\mu G(t, x(\sigma(t)))-\frac{\rho}{2} e(t)\right]\left(|x(t)|^{p-1} x(t)\right)^{-1} \\
& \geq {\left[\lambda f(t)|x(\tau(t))|^{p_{1}}+\frac{\rho}{2}|e(t)|\right]|x(t)|^{-p} } \\
&+\left[\mu g(t)|x(\sigma(t))|^{p_{2}}+\frac{\rho}{2}|e(t)|\right]|x(t)|^{-p} .
\end{aligned}
$$

Now, just the same as in the proofs of previous delay and advanced cases with $q>p$ and with the help of (104) in particular for

$$
\begin{aligned}
& \gamma=\frac{p_{1}}{p}>1, \quad X=(\lambda f(t))^{p / p_{1}}|x(\tau(t))|^{p}, \\
& Y=\left(\frac{p}{p_{1}-p} \frac{\rho}{2}|e(t)|\right)^{p / p_{1}}
\end{aligned}
$$

we have

$$
\begin{aligned}
& {\left[\lambda f(t)|x(\tau(t))|^{p_{1}}+\frac{\rho}{2|e(t)|}\right]|x(t)|^{-p} } \\
&=\left[X^{\gamma}+(\gamma-1) Y^{\gamma}\right]|x(t)|^{-p} \\
& \geq \frac{p_{1}}{p}(\lambda f(t))^{p / p_{1}}|x(\tau(t))|^{p} \\
& \times\left(\frac{p}{p_{1}-p} \frac{\rho}{2}|e(t)|\right)^{\left(p / p_{1}\right)\left(\left(p_{1} / p\right)-1\right)}|x(t)|^{-p} \\
&= \lambda^{p / p_{1}} \rho^{1-\left(p / p_{1}\right)} \frac{p_{1}}{p}\left(\frac{p}{2\left(p_{1}-p\right)}\right)^{1-\left(p / p_{1}\right)} \\
& \times|e(t)|^{1-\left(p / p_{1}\right)}(f(t))^{p / p_{1}}\left(\frac{x(\tau(t))}{x(t)}\right)^{p} \\
& \geq k(\lambda, \mu, \rho) \frac{p_{1}}{p}\left(\frac{p}{2\left(p_{1}-p\right)}\right)^{1-\left(p / p_{1}\right)} \\
& \times|e(t)|^{1-\left(p / p_{1}\right)}(f(t))^{p / p_{1}} \tau_{n}(t),
\end{aligned}
$$

where the function $k(\lambda, \mu, \rho)$ is from (24). Analogously, we show that

$$
\begin{gathered}
{\left[\mu g(t)|x(\sigma(t))|^{p_{2}}+\frac{\rho}{2}|e(t)|\right]|x(t)|^{-p}} \\
\geq k(\lambda, \mu, \rho) \frac{p_{2}}{p}\left(\frac{p}{2\left(p_{2}-p\right)}\right)^{1-\left(p / p_{2}\right)} \\
\quad \times|e(t)|^{1-\left(p / p_{2}\right)}(f(t))^{p / p_{2}} \sigma_{n}(t) .
\end{gathered}
$$


Summarizing previous calculation, we obtain

$$
\begin{aligned}
& {[\lambda F(t, x(\tau(t)))+\mu G(t, x(\sigma(t)))-\rho e(t)]} \\
& \quad \times\left(|x(t)|^{p-1} x(t)\right)^{-1} \geq k(\lambda, \mu, \rho) Q_{n}(t), \quad t \in J_{n},
\end{aligned}
$$

where the function $k(\lambda, \mu, \rho)$ is from (24).

(v) Supersublinear Delay-Advanced Case. Since $p_{1}>p>p_{2}$ and the following well-known elementary inequality holds

$$
\eta_{0} u_{0}+\eta_{1} u_{1}+\eta_{2} u_{2} \geq u_{0}^{\eta_{0}} u_{1}^{\eta_{1}} u_{2}^{\eta_{2}}, \quad \eta_{i} \geq 0, u_{i} \geq 0
$$

from $\eta_{0}, \eta_{1}, \eta_{2} \in(0,1), \eta_{0}+\eta_{1}+\eta_{2}=1$ and $p_{1} \eta_{1}+p_{2} \eta_{2}=p$, we obtain for all $t \in J_{n}$, for all $t \in J_{n}$ we obtain

$$
\begin{aligned}
& {[\lambda F(t, x(\tau(t)))+\mu G(t, x(\sigma(t)))-\rho e(t)]} \\
& \times\left(|x(t)|^{p-1} x(t)\right)^{-1} \\
& \geq\left[\lambda f(t)|x(\tau(t))|^{p_{1}}+\mu g(t)|x(\sigma(t))|^{p_{2}}+\rho|e(t)|\right] \\
& \times|x(t)|^{-p} \\
& =\left[\eta_{1}\left[\eta_{1}^{-1} \lambda f(t)|x(\tau(t))|^{p_{1}}\right]+\eta_{2}\left[\eta_{2}^{-1} \mu g(t)|x(\sigma(t))|^{p_{2}}\right]\right. \\
& \left.+\eta_{0}\left[\eta_{0}^{-1} \rho|e(t)|\right]\right]|x(t)|^{-p} \\
& \geq\left[\eta_{1}^{-1} \lambda f(t)|x(\tau(t))|^{p_{1}}\right]^{\eta_{1}}\left[\eta_{2}^{-1} \mu g(t)|x(\sigma(t))|^{p_{2}}\right]^{\eta_{2}} \\
& \times\left[\eta_{0}^{-1} \rho|e(t)|\right]^{\eta_{0}}|x(t)|^{-p} \\
& =\lambda^{\eta_{1}} \mu^{\eta_{2}} \rho^{\eta_{0}}|e(t)|^{\eta_{0}}(f(t))^{\eta_{1}}(g(t))^{\eta_{2}} \\
& \times \frac{|x(\tau(t))|^{\eta_{1} p_{1}}}{|x(t)|^{\eta_{1} p_{1}}} \frac{|x(\sigma(t))|^{\eta_{2} p_{2}}}{|x(t)|^{\eta_{2} p_{2}}} \prod_{i=0}^{2} \eta_{i}^{-\eta_{i}} \\
& =k(\lambda, \mu, \rho)|e(t)|^{\eta_{0}}(f(t))^{\eta_{1}}(g(t))^{\eta_{2}} \\
& \times\left(\frac{x(\tau(t))}{x(t)}\right)^{\eta_{1} p_{1}}\left(\frac{x(\sigma(t))}{x(t)}\right)^{\eta_{2} p_{2}} \prod_{i=0}^{2} \eta_{i}^{-\eta_{i}} \\
& \geq k(\lambda, \mu, \rho)|e(t)|^{\eta_{0}}(f(t))^{\eta_{1}}(g(t))^{\eta_{2}}\left(\tau_{n}(t)\right)^{\eta_{1}\left(p_{1} / p\right)} \\
& \times\left(\sigma_{n}(t)\right)^{\eta_{2}\left(p_{2} / p\right)} \prod_{i=0}^{2} \eta_{i}^{-\eta_{i}} \\
& =k(\lambda, \mu, \rho) Q_{n}(t),
\end{aligned}
$$

where $k(\lambda, \mu, \rho)$ and $Q_{n}(t)$ are given, respectively, in (24) and (25). Thus, it is shown that required condition (5) in the cases (i)-(iv) is fulfilled with respect to $k(\lambda, \mu, \rho)$ and $Q_{n}(t)$ determined by (18), (21), or (24) and (19), (22), or (25).

In conclusion, according to the previous observation, we see that all assumptions of Lemma 4 are fulfilled and, hence, Lemma 4 proves Theorems 5, 6, and 7 .

\section{Conflict of Interests}

The author declares that there is no conflict of interests regarding the publication of this paper.

\section{References}

[1] Y. G. Sun, "A note on Nasr's and Wong's papers," Journal of Mathematical Analysis and Applications, vol. 286, no. 1, pp. 363367, 2003.

[2] Y. G. Sun, C. H. Ou, and J. S. W. Wong, "Interval oscillation theorems for a second-order linear differential equation," Computers \& Mathematics with Applications, vol. 48, no. 10-11, pp. 1693-1699, 2004.

[3] S. Murugadass, E. Thandapani, and S. Pinelas, "Oscillation criteria for forced second-order mixed type quasilinear delay differential equations," Electronic Journal of Differential Equations, vol. 2010, article 73, 9 pages, 2010.

[4] Y. Bai and L. Liu, "New oscillation criteria for second-order delay differential equations with mixed nonlinearities," Discrete Dynamics in Nature and Society, vol. 2010, Article ID 796256, 9 pages, 2010.

[5] A. F. Güvenilir and A. Zafer, "Second-order oscillation of forced functional differential equations with oscillatory potentials," Computers \& Mathematics with Applications, vol. 51, no. 9-10, pp. 1395-1404, 2006.

[6] A. Zafer, "Interval oscillation criteria for second order superhalf linear functional differential equations with delay and advanced arguments," Mathematische Nachrichten, vol. 282, no. 9, pp. 1334-1341, 2009.

[7] A. F. Guvenilir, "Interval oscillation of second-order functional differential equations with oscillatory potentials," Nonlinear Analysis: Theory, Methods \& Applications, vol. 71, no. 12, pp. e2849-e2854, 2009.

[8] T. S. Hassan, L. Erbe, and A. Peterson, "Forced oscillation of second order differential equations with mixed nonlinearities," Acta Mathematica Scientia B, vol. 31, no. 2, pp. 613-626, 2011.

[9] M. Pašić, "New oscillation criteria for second-order forced quasilinear functional differential equations," Abstract and Applied Analysis, vol. 2013, Article ID 735360, 12 pages, 2013.

[10] L. H. Erbe, Q. Kong, and B. G. Zhang, Oscillation Theory for Functional-Differential Equations, vol. 190, Marcel Dekker, New York, NY, USA, 1995.

[11] V. Kolmanovskii and A. Myshkis, Introduction to the Theory and Applications of Functional-Differential Equations, vol. 463, Kluwer Academic Publishers, Dordrecht, The Netherlands, 1999.

[12] R. P. Agarwal, M. Bohner, and W.-T. Li, Nonoscillation and Oscillation: Theory for Functional Differential Equations, vol. 267, Marcel Dekker, New York, NY, USA, 2004.

[13] L. Erbe, T. Hassan, and A. Peterson, "Oscillation of second order functional dynamic equations," International Journal of Difference Equations, vol. 5, no. 2, pp. 175-193, 2010.

[14] B. Baculíková, J. Džurina, and Y. V. Rogovchenko, "Oscillation of third order trinomial delay differential equations," Applied Mathematics and Computation, vol. 218, no. 13, pp. 7023-7033, 2012.

[15] R. P. Agarwal, L. Berezansky, E. Braverman, and A. Domoshnitsky, Nonoscillation Theory of Functional Differential Equations with Applications, Springer, New York, NY, USA, 2012. 
[16] J. Zhang, "Variational approach to solitary wave solution of the generalized Zakharov equation," Computers \& Mathematics with Applications, vol. 54, no. 7-8, pp. 1043-1046, 2007.

[17] T. Öziş and A. Yıldırım, "Application of He's semi-inverse method to the nonlinear Schrödinger equation," Computers \& Mathematics with Applications, vol. 54, no. 7-8, pp. 1039-1042, 2007.

[18] X.-C. Cai and M.-S. Li, "Periodic solution of Jacobi elliptic equations by He's perturbation method," Computers \& Mathematics with Applications, vol. 54, no. 7-8, pp. 1210-1212, 2007.

[19] S. Lenci, G. Menditto, and A. M. Tarantino, "Homoclinic and heteroclinic bifurcations in the non-linear dynamics of a beam resting on an elastic substrate," International Journal of NonLinear Mechanics, vol. 34, no. 4, pp. 615-632, 1999.

[20] D.-J. Huang and H.-Q. Zhang, "Link between travelling waves and first order nonlinear ordinary differential equation with a sixth-degree nonlinear term," Chaos, Solitons \& Fractals, vol. 29, no. 4, pp. 928-941, 2006.

[21] A. I. Maimistov, "Propagation of an ultimately short electromagnetic pulse in a nonlinear medium described by the fifthorder Duffing model," Optics and Spectroscopy, vol. 94, pp. 251257, 2003.

[22] M. N. Hamdan and N. H. Shabaneh, "On the large amplitude free vibrations of a restrained uniform beam carrying an intermediate lumped mass," Journal of Sound and Vibration, vol. 199, no. 5, pp. 711-736, 1997.

[23] J.-H. He, "Some asymptotic methods for strongly nonlinear equations," International Journal of Modern Physics B, vol. 20, no. 10, pp. 1141-1199, 2006.

[24] J. B. Marion, Classical Dynamics of Particles and Systems, 1970.

[25] I. Kovacic and M. J. Brennan, The Duffing Equation: Nonlinear Oscillatos and their Behaviour, John Wiley \& Sons, 1st edition, 2011.

[26] F. C. Moon, Chaotic Vibrations: An Introduction for Applied Scientists and Engineers, John Wiley \& Sons, New York, NY, USA, 2004.

[27] J. J. Stoker, Nonlinear Vibrations, 1950.

[28] G. Chen and Z. Tao, "Amplitude-frequency relationship for the relativistic oscillator," AASRI Procedia, vol. 1, pp. 400-403, 2012.

[29] R. E. Mickens, Oscillations in Planar Dynamic Systems, World Scientific Publishing, Singapore, 1996.

[30] A. Belendez, T. Belendez, C. Neipp, A. Hernandez, and M. L. Alvarez, "Approximate solutions of a nonlinear oscillator typified as a mass attached to a stretched elastic wire by the homotopy perturbation method," Chaos, Solitions and Fractals, vol. 39, pp. 746-764, 2009.

[31] A. Belendez, E. Fernandez, R. Fuentes, J. J. Rodes, and I. Pascual, "Harmonic balancing approach to nonlinear oscillations of a punctual charge in the eletric field of charged ring," Physics Letters A, vol. 373, pp. 735-740, 2009.

[32] A. Elías-Zúñiga, "Exact solution of the cubic-quintic Duffing oscillator," Applied Mathematical Modelling, vol. 37, no. 4, pp. 2574-2579, 2013.

[33] A. Beléndez, M. L. Alvarez, J. Francés et al., "Analytical approximate solutions for the cubic-quintic Duffing oscillator in terms of elementary functions," Journal of Applied Mathematics, vol. 2012, Article ID 286290, 16 pages, 2012.

[34] A. Elías-Zúñiga, O. Martínez-Romero, and R. K. Córdoba-Díaz, "Approximate solution for the Duffing-harmonic oscillator by the enhanced cubication method," Mathematical Problems in Engineering, vol. 2012, Article ID 618750, 12 pages, 2012.
[35] C. W. Lim, B. S. Wu, and W. P. Sun, "Higher accuracy analytical approximations to the Duffing-harmonic oscillator," Journal of Sound and Vibration, vol. 296, no. 4-5, pp. 1039-1045, 2006.

[36] J. He, "Some new approaches to Duffing equation with strongly and high order nonlinearity. II: parametrized perturbation technique," Communications in Nonlinear Science \& Numerical Simulation, vol. 4, no. 1, pp. 81-83, 1999.

[37] V. Marinca and N. Herisanu, "Periodic solutions for some strongly nonlinear oscillations by He's variational iteration method," Computers \& Mathematics with Applications, vol. 54, no. 7-8, pp. 1188-1196, 2007.

[38] W. Lu and Y. Liu, "Vibration control for the primary resonance of the Duffing oscillator by a time delay state feedback," International Journal of Nonlinear Science, vol. 8, no. 3, pp. 324328, 2009.

[39] H. Y. Hu and Z. H. Wang, Dynamics of Controlled Mechanical Systems with Delayed Feedback, Springer, 2002.

[40] M. Hamdi and M. Belhaq, "Control of bistability in a delayed Duffing oscillator," Advances in Acoustics and Vibration, vol. 2012, Article ID 872498, 6 pages, 2012.

[41] V. Ravichandran, C. Chinnathambi, and S. Rajasekar, "Nonlinear resonance in Duffing oscillator with fixed and integrative time-delayed feedbacks," Pramana Journal of Physics, vol. 78, pp. 347-360, 2013.

[42] X. You and H. Xu, "Analytical approximations for the periodic motion of the Duffing system with delayed feedback," Numerical Algorithms, vol. 56, no. 4, pp. 561-576, 2011.

[43] Z. Sun, W. Xu, X. Yang, and T. Fang, "Inducing or suppressing chaos in a double-well Duffing oscillator by time delay feedback," Chaos, Solitons and Fractals, vol. 27, pp. 705-714, 2006.

[44] H. Wang, H. Hu, and Z. Wang, "Global dynamics of a Duffing oscillator with delayed displacement feedback," International Journal of Bifurcation and Chaos in Applied Sciences and Engineering, vol. 14, no. 8, pp. 2753-2775, 2004.

[45] J. Chiasson and J. J. Loiseau, Applications of Time Delay Systems, Springer, 2007.

[46] M. Lakshmanan and D. V. Senthilkumar, Dynamics of Nonlinear Time-Delay Systems, Springer, 2010.

[47] G. Stépán, T. Insperger, and R. Szalai, "Delay, parametric excitation, and the nonlinear dynamics of cutting processes," International Journal of Bifurcation and Chaos in Applied Sciences and Engineering, vol. 15, no. 9, pp. 2783-2798, 2005.

[48] U. van der Heiden and H.-O. Walther, "Existence of chaos in control systems with delayed feedback," Journal of Differential Equations, vol. 47, no. 2, pp. 273-295, 1983.

[49] Y. G. Sun and J. S. W. Wong, "Oscillation criteria for second order forced ordinary differential equations with mixed nonlinearities," Journal of Mathematical Analysis and Applications, vol. 334, no. 1, pp. 549-560, 2007.

[50] J. Heagy and W. L. Ditto, "Dynamics of a two-frequency parametrically driven Duffing oscillator," Journal of Nonlinear Science, vol. 1, no. 4, pp. 423-455, 1991.

[51] A. B. Belogortsev, "Bifurcations of tori and chaos in the quasiperiodically forced Duffing oscillator," Nonlinearity, vol. 5, no. 4, pp. 889-897, 1992.

[52] M. Belhaq and M. Houssni, "Quasi-periodic oscillations, chaos and suppression of chaos in a nonlinear oscillator driven by parametric and external excitations," Nonlinear Dynamics, vol. 18, no. 1, pp. 1-24, 1999.

[53] S. H. Saker, P. Y. H. Pang, and R. P. Agarwal, "Oscillation theorems for second order nonlinear functional differential 
equations with damping," Dynamic Systems and Applications, vol. 12, no. 3-4, pp. 307-321, 2003.

[54] I. N. Bronshtein, K. A. Semendyayev, G. Musiol, and H. Muehlig, Handbook of Mathematics, Springer, 5th edition, 2007.

[55] M. Pašić, "Fite-Wintner-Leighton-type oscillation criteria for second-order differential equations with nonlinear damping," Abstract and Applied Analysis, vol. 2013, Article ID 852180, 10 pages, 2013. 


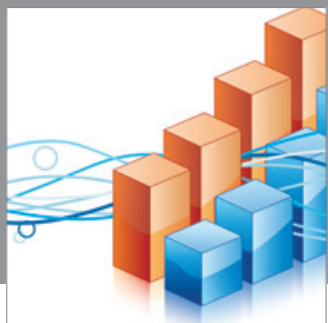

Advances in

Operations Research

mansans

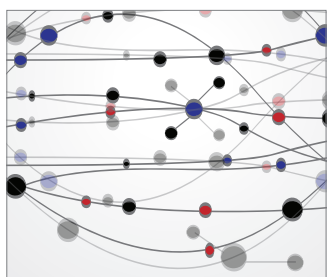

The Scientific World Journal
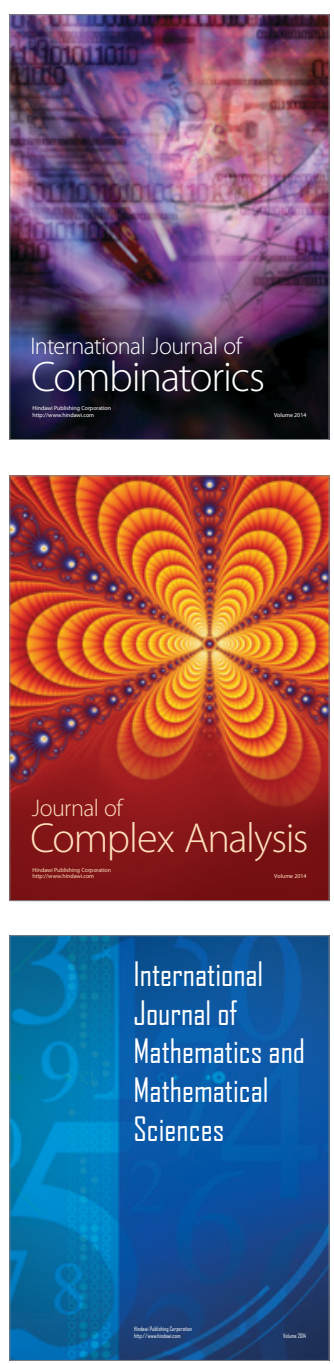
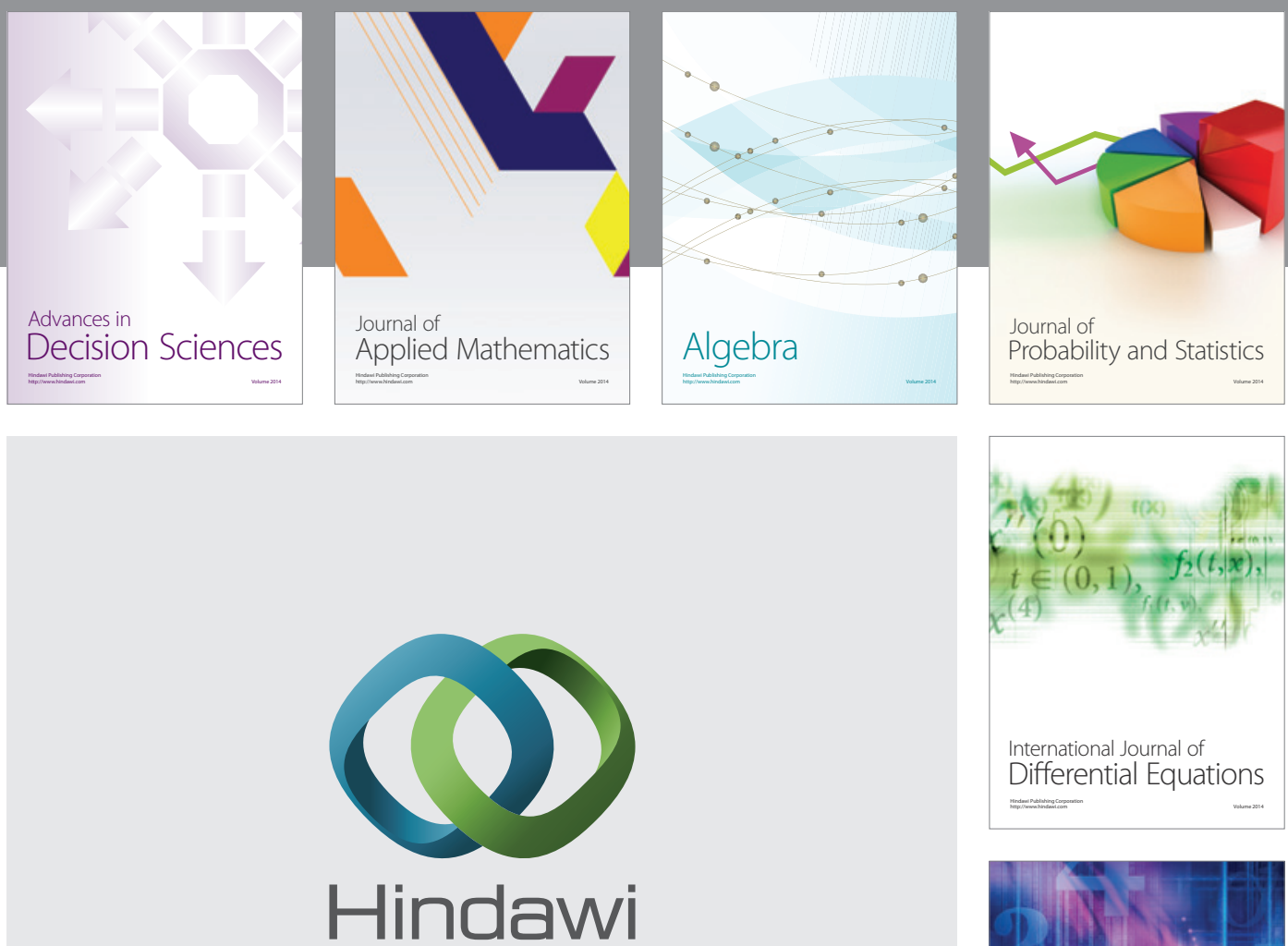

Submit your manuscripts at http://www.hindawi.com
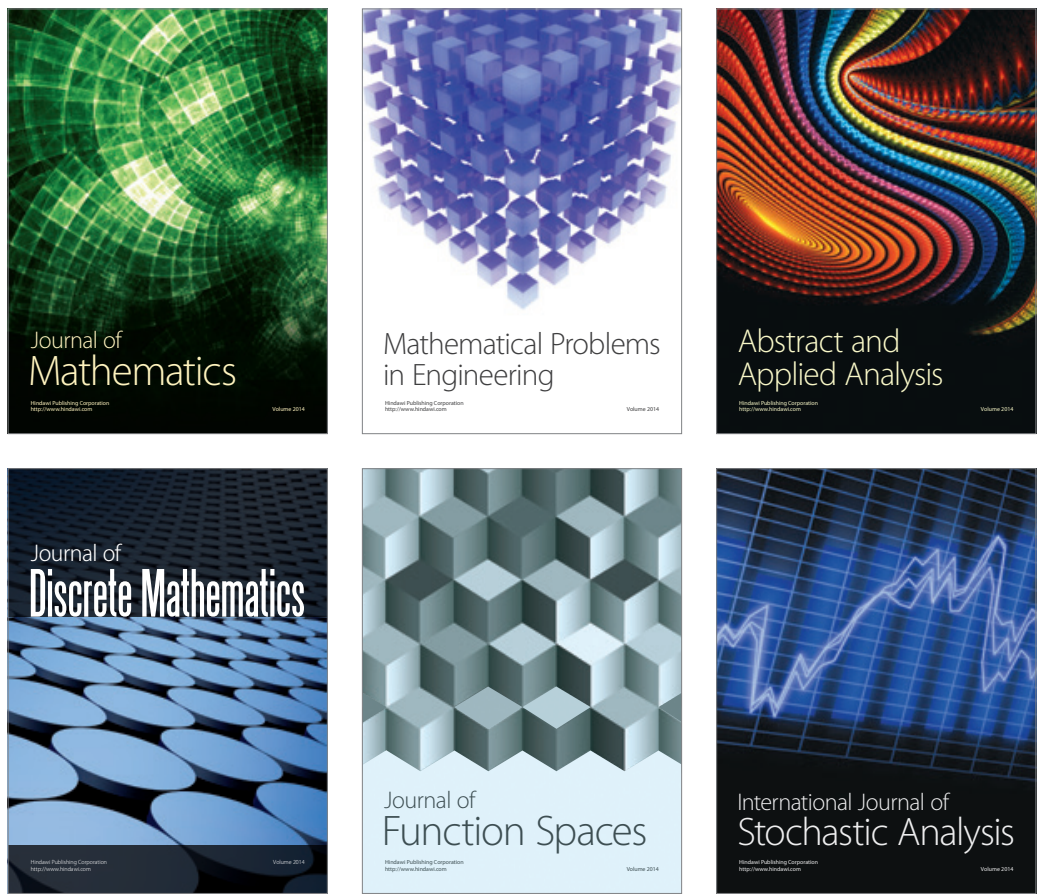

Journal of

Function Spaces

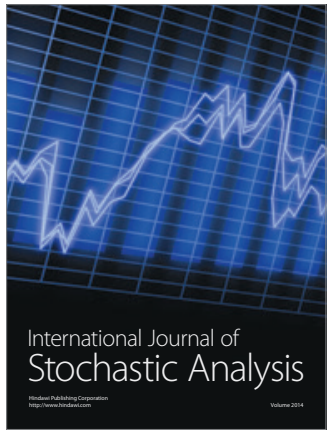

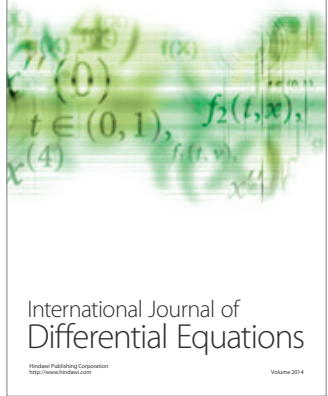
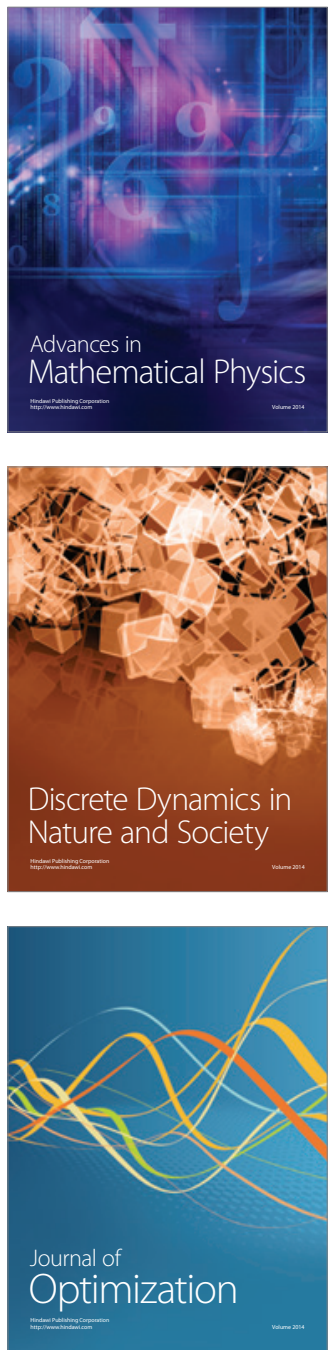